\title{
Density functional simulation of spontaneous formation of vesicle in block copolymer solutions
}

\author{
AUTHOR(S): \\ Uneyama, $\mathrm{T}$
}

\section{CITATION:}

Uneyama, T. Density functional simulation of spontaneous formation of vesicle in block copolymer solutions. JOURNAL OF CHEMICAL PHYSICS 2007, 126(11): 114902.

\section{ISSUE DATE:}

2007-03-21

URL:

http://hdl.handle.net/2433/49939

\section{RIGHT:}

Copyright 2007 American Institute of Physics. This article may be downloaded for personal use only. Any other use requires prior permission of the author and the American Institute of Physics. 


\title{
Density functional simulation of spontaneous formation of vesicle in block copolymer solutions
}

\author{
Takashi Uneyama \\ Department of Physics, Graduate School of Science, Kyoto University, Sakyo-ku, Kyoto 606-8502, Japan
}

(Received 22 June 2006; accepted 9 January 2007; published online 16 March 2007)

\begin{abstract}
The author carries out numerical simulations of vesicle formation based on the density functional theory for block copolymer solutions. It is shown by solving the time evolution equations for concentrations that a polymer vesicle is spontaneously formed from the homogeneous state. The vesicle formation mechanism obtained by this simulation agrees with the results of other simulations based on the particle models as well as experiments. By changing parameters such as the volume fraction of polymers or the Flory-Huggins interaction parameter between the hydrophobic subchains and solvents, the spherical micelles, cylindrical micelles, or bilayer structures can also be obtained. The author also shows that the morphological transition dynamics of the micellar structures can be reproduced by controlling the Flory-Huggins interaction parameter. (C) 2007 American Institute of Physics. [DOI: 10.1063/1.2463426]
\end{abstract}

\section{INTRODUCTION}

Amphiphilic block copolymers, which consist of hydrophilic subchains and hydrophobic subchains, are known to form micellar structures, such as spherical micelles, cylindrical micelles, and vesicles. ${ }^{1,2}$ To clarify how these structures are self-organized is a basic problem of the kinetics of micellar systems.

Several computer simulations have been available to study the formation of a vesicle based on the particle models [Brownian dynamics (BD), ${ }^{3}$ dissipative particle dynamics (DPD), ${ }^{4}$ and molecular dynamics ${ }^{5}$ (MD)]. The spontaneous vesicle formation process from the homogeneous state observed in these simulations is as follows. The amphiphilic block copolymers aggregate into small spherical micelles rapidly from the homogeneous initial state. The spherical micelles grow to larger micelles by collision (cylindrical micelles, open disklike micelles). The large disklike micelles finally close up and form vesicles. The micelle growth process and the closure process are slower than the first spherical micelle formation process. Hereafter, this process will be called "mechanism I" [see also Fig. 1(a)]. Note that mechanism I is also supported by Monte Carlo simulations ${ }^{6}$ or experiments of lipid systems. ${ }^{7,8}$ Strictly speaking, the mechanism obtained by the experiments for lipid systems may be different from the one for polymer systems. However, since the DPD simulations for polymer systems and the BD and MD simulations for lipid systems give similar results (mechanism I), it is believed that the vesicle formation mechanism is common for polymer systems and lipid systems. Also noted is the similar mechanism obtained for polymer systems by the experiments of morphological transition of cylindrical micelles to vesicles. ${ }^{9}$

The field theoretical approach, which has been developed to study mesoscale structures of block copolymers, ${ }^{10-16}$ is considered to be useful for vesicle formation, but most of the works have been limited to simulations in thermal equilibrium. ${ }^{17,18}$ Since these simulations ignore realistic ki- netics (for example, local mass conservation is not satisfied), the vesicle formation process observed in these simulations is different from mechanism I. The first process is similar to mechanism I. Small spherical micelles are formed rapidly. The spherical micelles then grow up to large spherical micelles by the evaporation-condensation-like process. The large spherical micelles are energetically unfavorable, and thus the large micelles take the solvents into them to lower the energy. This process is called "mechanism II" [see also Figure 1(b)].

The dynamical simulations for diblock copolymer solutions were carried by Sevink and Zvelindovsky, ${ }^{19}$ using the dynamic self-consistent field (SCF) theory. However, in their simulations both subchains are hydrophobic and the resulting structures are so-called onion structures. ${ }^{19-22}$ The onion structures obtained by simulations are essentially microphase separation structures in the block copolymer rich droplets in the solvents and are qualitatively different from the multilayer vesicle structures, which are often called "onion structures" in surfactant solutions. The vesicle structures contain solvents inside them and are therefore different from

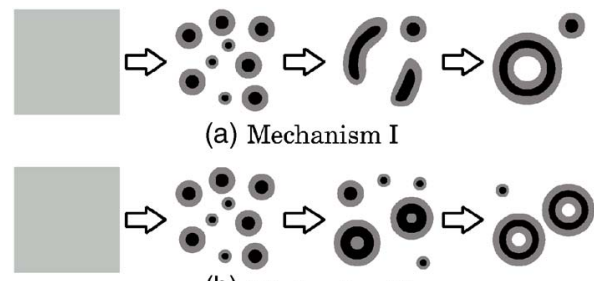

(b) Mechanism II

FIG. 1. Schematic representation of two vesicle formation mechanisms from the initial homogeneous state. Black and gray color correspond to hydrophobic and hydrophilic subchains, respectively. (a) Mechanism I: first, small micellar structures are formed. The micellar structures grow by collision and become large cylindrical or open disklike micelles. Finally, the open disklike micelles close up to form closed vesicles. (b) Mechanism II: the initial stage is similar to mechanism I. However, the small micellar structures formed in the initial stage grow up to be large spherical micelles. The large spherical micelles then take the solvent inside and thus vesicles are formed. 
polymer onion structures, and one should distinguish the onion formation process from the vesicle formation process. Also note that the simulations for onion structures were carried out for a weak segregation region and this does not agree with previous equilibrium simulations for vesicles, since vesicles are observed in a rather strong segregation region. (In this work, "strong segregation region" denotes as the region where the minimum and maximum values of the density field are approximately 0 and 1 . One may call such a region the intermediate segregation region.) It should be noted that the formation processes of the onion structures by simulations ${ }^{19,21}$ are mainly the lamellar ordering process in dropletlike regions. Therefore the resulting morphologies (onions) are similar to the phase separation patterns in droplets $^{23}$ than vesicles. Thus, the onion formation process is not considered mechanism I.

Recently, dynamical simulations of vesicle formation have been done by He and Schmid, ${ }^{24}$ using the external potential dynamics (EPD). ${ }^{25}$ They obtained vesicles, but their formation process is similar to mechanism II. Unfortunately mechanism II is qualitatively different from mechanism I, and this means that their result does not agree with particle simulations or experiments (the reason for this difference will be discussed in Sec. IV B). It is expected that the mechanisms observed by different simulation methods should be the same.

In the present work, the previous model, density functional theory for block copolymers, ${ }^{18,22}$ will be applied to the dynamics. Numerical simulations for amphiphilic diblock copolymer solutions will be carried out in three dimensions. By using the continuous field model, it is shown that a vesicle is spontaneously formed from a disordered uniform phase. The simulation result is consistent with mechanism I. It is shown that the spontaneous formation process of various micellar structures (such as spherical micelles or cylindrical micelles) and the morphological transition dynamics can be simulated.

\section{THEORY}

The dynamics of block copolymer systems are well studied by using the dynamic SCF theory ${ }^{15}$ as well as the timedependent Ginzburg-Landau (TDGL) equation. ${ }^{26}$ The dynamic SCF simulations are known to be accurate from the weak segregation region to strong segregation region, but they consume memory and need large CPU power. In contrast, the TDGL simulations need less CPU power and enables large scale simulations. However, the free energy functionals ${ }^{10,11}$ used in the TGDL approach are generally not appropriate for the strong segregation region, since the validity of the Ginzburg-Landau (GL) expansion is guaranteed only for the weak segregation region where the density fluctuation is sufficiently small. ${ }^{14,22,27}$ Actually Maurits and Fraaije $^{28}$ showed that the widely used fourth-order GL expansion model is not sufficient for dynamical simulations. To overcome this limitation it is necessary to use free energy functional model such as the Flory-Huggins-de GennesLifshitz free energy, ${ }^{29-31}$ which is not expressed in the GL expansion form.
In previous simulations, vesicles are observed for rather strong segregation region in diblock copolymer solutions. Thus one can conclude that the use of inappropriate free energy functional models qualitatively affects micellar structures in diblock copolymer solutions. It is necessary to use a free energy model and a dynamic equation which is valid for the strong segregation region and for the macrophase separation. In a previous work, ${ }^{22}$ the free energy functional, which is valid for strong segregation region, that is, valid for vesicles was proposed. ${ }^{18}$

\section{A. Free energy functional}

The free energy functional for the system can be expressed as follows by using the soft-colloid picture: ${ }^{31-33}$

$$
F=-T S+U=-T S_{\text {trans }}-T S_{\text {conf }}+U,
$$

where $S$ and $U$ are the entropy functional and the interaction energy functional, and $T$ is the temperature. The entropy functional can be decomposed into two parts: the translational entropy functional $S_{\text {trans }}$ and the conformational entropy functional $S_{\text {conf }}$. In the density functional theory, $U$, $S_{\text {trans }}$, and $S_{\text {conf }}$ are expressed as the functional of the local volume fraction fields. (The local volume fraction field is equivalent to the local density field, if the segment volume is set to unity. It is assumed that the segment volume is unity in this work.)

Each contribution to the free energy functional can be modeled for $A B$-type diblock copolymer and solvent mixtures by ${ }^{18,22}$

$$
\begin{aligned}
-\frac{S_{\text {trans }}}{k_{B}}= & \sum_{i(=A, B)} \int d \boldsymbol{r} f_{i} C_{i i} \phi_{i}(\boldsymbol{r}) \ln \phi_{i}(\boldsymbol{r}) \\
& +\int d \boldsymbol{r} \phi_{S}(\boldsymbol{r}) \ln \phi_{S}(\boldsymbol{r}),
\end{aligned}
$$

$$
\begin{aligned}
&-\frac{S_{\mathrm{conf}}}{k_{B}}= \sum_{i, j=A, B)} \int d \boldsymbol{r} d \boldsymbol{r}^{\prime} 2 \sqrt{f_{i} f_{j}} A_{i j} \tilde{\mathcal{G}}\left(\boldsymbol{r}-\boldsymbol{r}^{\prime}\right) \psi_{i}(\boldsymbol{r}) \psi_{j}\left(\boldsymbol{r}^{\prime}\right) \\
&+\int d \boldsymbol{r} 4 \sqrt{f_{A} f_{B}} C_{A B} \psi_{A}(\boldsymbol{r}) \psi_{B}(\boldsymbol{r}) \\
&+\sum_{i(=A, B, S)} \int d \boldsymbol{r} \frac{b^{2}}{6}\left|\nabla \psi_{i}(\boldsymbol{r})\right|^{2}, \\
& \frac{U}{k_{B} T}=\sum_{i, j(=A, B, S)} \int d \boldsymbol{r} \frac{\chi_{i j}}{2} \phi_{i}(\boldsymbol{r}) \phi_{j}(\boldsymbol{r})+\int d \boldsymbol{r} \frac{P(\boldsymbol{r})}{2}\left[\phi_{A}(\boldsymbol{r})\right. \\
&\left.+\phi_{B}(\boldsymbol{r})+\phi_{S}(\boldsymbol{r})-1\right],
\end{aligned}
$$

where $k_{B}$ is the Boltzmann constant, $\phi_{i}(\boldsymbol{r})$ is the local volume fraction of the $i$ segment at position $\boldsymbol{r}, \psi_{i}(\boldsymbol{r})$ is the order parameter field ( $\psi$ field) defined as $\psi_{i}(\boldsymbol{r}) \equiv \sqrt{\phi_{i}(\boldsymbol{r}){ }^{31}}$ The coefficients $A_{i j}$ and $C_{i j}$ are constants determined from the block copolymer architecture (the explicit forms of $A_{i j}$ and $C_{i j}$ are not shown here; they can be found in Refs. 18 and 22), $f_{i}$ is the block ratio of the block copolymer, $b$ is the Kuhn length, $\chi_{i j}$ is the Flory-Huggins $\chi$ parameter, and $\widetilde{\mathcal{G}}\left(\boldsymbol{r}-\boldsymbol{r}^{\prime}\right)$ is the Green function which satisfies $\left[-\nabla^{2}+\lambda^{-2}\right] \widetilde{\mathcal{G}}\left(\boldsymbol{r}-\boldsymbol{r}^{\prime}\right)$ 
$=\delta\left(\boldsymbol{r}-\boldsymbol{r}^{\prime}\right),{ }^{34,35}$ where $\lambda$ is a cutoff length and is about the size of microphase separation structures. $P(\boldsymbol{r})$ is the Lagrange multiplier for the incompressible condition $\left(\phi_{A}(\boldsymbol{r})+\phi_{B}(\boldsymbol{r})\right.$ $\left.+\phi_{S}(\boldsymbol{r})=1\right)^{36}$

The conformational entropy [Eq. (3)] is expressed in the bilinear form of $\psi$ field. ${ }^{22,30,37}$ One significant property of the conformational entropy is that it satisfies the following relation:

$$
S_{\text {cont }}\left[\left\{\alpha \phi_{i}(\boldsymbol{r})\right\}\right]=\alpha S_{\text {cont }}\left[\left\{\phi_{i}(\boldsymbol{r})\right\}\right],
$$

where $\alpha$ is an arbitrary positive constant. [It is clear that the conformational entropy in the bilinear form of $\psi$ satisfies Eq. (5)]. Equation (5) can be interpreted as the extensivity of the conformational entropy; under the current approximation (using mean field and neglecting many-body correlations), the total conformational entropy is the sum of the conformational entropy of each polymer chain. Since the density profiles of micellar structures are determined to achieve the low free energy, the conformational entropy which does not satisfy Eq. (5) may lead to the unphysical density profile. Note that the Lifshitz entropy ${ }^{30,37}$ and the conformational entropy calculated by the SCF theory satisfy Eq. (5), while most of the previous phenomenological free energy models do not satisfy Eq. (5).

Substituting Eqs. (2)-(4) into Eq. (1), yields

$$
\begin{aligned}
\frac{F}{k_{B} T}= & \sum_{i(=A, B)} \int d \boldsymbol{r} 2 f_{i} C_{i i} \psi_{i}^{2}(\boldsymbol{r}) \ln \psi_{i}(\boldsymbol{r}) \\
& +\int d \boldsymbol{r} 2 \psi_{S}^{2}(\boldsymbol{r}) \ln \psi_{S}(\boldsymbol{r}) \\
& +\sum_{i, j(=A, B)} \int d \boldsymbol{r} d \boldsymbol{r}^{\prime} 2 \sqrt{f_{i} f_{j}} A_{i j} \widetilde{\mathcal{G}}\left(\boldsymbol{r}-\boldsymbol{r}^{\prime}\right) \psi_{i}(\boldsymbol{r}) \psi_{j}\left(\boldsymbol{r}^{\prime}\right) \\
& +\int d \boldsymbol{r} 4 \sqrt{f_{A} f_{B}} C_{A B} \psi_{A}(\boldsymbol{r}) \psi_{B}(\boldsymbol{r}) \\
& +\sum_{i(=A, B, S)} \int d \boldsymbol{r} \frac{b^{2}}{6}\left|\nabla \psi_{i}(\boldsymbol{r})\right|^{2} \\
& +\sum_{i, j(=A, B, S)} \int d \boldsymbol{r} \frac{\chi_{i j}}{2} \psi_{i}^{2}(\boldsymbol{r}) \psi_{j}^{2}(\boldsymbol{r}) \\
& +\int d \boldsymbol{r} \frac{P(\boldsymbol{r})}{2}\left[\psi_{A}^{2}(\boldsymbol{r})+\psi_{B}^{2}(\boldsymbol{r})+\psi_{S}^{2}(\boldsymbol{r})-1\right] .
\end{aligned}
$$

The difference between the free energy functional [Eq. (6)] and a previous model ${ }^{22}$ is the form of the Green function [in the previous theory, the Green function $\mathcal{G}\left(\boldsymbol{r}-\boldsymbol{r}^{\prime}\right)$ has no cutoff, $\left.-\nabla^{2} \mathcal{G}\left(\boldsymbol{r}-\boldsymbol{r}^{\prime}\right)=\delta\left(\boldsymbol{r}-\boldsymbol{r}^{\prime}\right)\right]$. A cutoff for the Green function was first introduced by Tang and Freed, ${ }^{34}$ without derivation, for the Ohta-Kawasaki type standard GL free energy. ${ }^{11}$ The Ohta-Kawasaki type Coulomb type long range interaction term may cause unphysical interaction or correlation in nonperiodic systems such as block copolymer solutions. The true form of long range interaction can be, in principle, obtained by calculating higher order terms in free energy functional (the effect of higher order vertex functions) exactly. While the higher order terms can be calculated numerically by us- ing the SCF, it is practically impossible to get the analytical form of them and take a summation.

To avoid the unphysical correlation without numerically demanding calculations, in the present study, a theory is employed that describes more precisely the properties of the length scale of the order of one polymer chain. ${ }^{35}$ The Coulomb-type interaction is modified by introducing the Tang-Freed-type cutoff, ${ }^{34}$ instead of calculating higher order terms exactly. It is reasonable to consider that the interaction range (or the cutoff length $\lambda$ ) of the long range interaction cannot exceed the characteristic size of the polymer chain. Generally, it is difficult to determine the cutoff length (one way to determine it is to use the SCF calculation or other microscopic calculations such as the MD).

Here, the cutoff length is estimated for the simplest case, diblock copolymer melts. For homogeneous ideal state, the cutoff length is considered to be the mean square end to end distance of a polymer chain $N^{1 / 2} b$, where $N$ is the polymerization index. On the other hand, for microphase separation structures at the strong segregation region, the polymer chains are strongly stretched. The periods of the structures are known to be proportional to $N^{2 / 3} b .^{11,38}$ Thus the following polymerization index dependence for the cutoff length of diblock copolymer melts is acquired:

$$
\lambda \propto \begin{cases}N^{1 / 2} b & \text { (for ideal state) } \\ N^{2 / 3} b & \text { (for strong segregation region). }\end{cases}
$$

It is assumed that the micellar structures have a cutoff length similar to that of the melt case. In this work $\lambda \simeq N^{2 / 3} b$ is used for micellar systems. Note that this is a rough estimation and the validity is not guaranteed. For example, the proportional coefficient in Eq. (7) is ignored here, or the effect of swelling of the hydrophilic subchain in the solution is not taken into account. Therefore the validity of this value of $\lambda$ should be tested by comparing with the characteristic scale of the resulting phase separation structure. Also note that the dependence of the morphologies to the value of $\lambda$ is not so sensitive (see also Appendix C). What is important here is that the interaction range is not infinite (as the original OhtaKawasaki Green function) but finite (as the modified Green function by Tang and Freed).

\section{B. Dynamic equation}

The stochastic dynamic density functional model ${ }^{39-41}$ is employed for the time evolution equation. The diblock copolymer solutions are expressed as three component systems (hydrophilic subchain $A$, hydrophobic subchain $B$, and solvent $S$ ). The dynamic equation for multicomponent systems can be expressed as follows:

$$
\frac{\partial \phi_{i}(\boldsymbol{r})}{\partial t}=\nabla \cdot\left[\frac{1}{\zeta_{i}} \phi_{i}(\boldsymbol{r}) \nabla \frac{\delta F}{\delta \phi_{i}(\boldsymbol{r})}\right]+\xi_{i}(\boldsymbol{r}, t),
$$

where $\phi_{i}(\boldsymbol{r})$ is the concentration of $i$ th component $(i$ $=A, B, S)$ and $\zeta_{i}$ is a friction coefficient. $F$ is the free energy. $\xi_{i}(\boldsymbol{r}, t)$ is the thermal noise which satisfies the fluctuationdissipation relation.

$$
\left\langle\xi_{i}(\boldsymbol{r}, t)\right\rangle=0,
$$




$$
\begin{aligned}
\left\langle\xi_{i}(\boldsymbol{r}, t) \xi_{j}\left(\boldsymbol{r}^{\prime}, t^{\prime}\right)\right\rangle= & -\frac{2}{\zeta_{i}} \widetilde{\beta}^{-1} k_{B} T \delta_{i j} \nabla \cdot\left[\phi_{i}(\boldsymbol{r}) \nabla \delta\left(\boldsymbol{r}-\boldsymbol{r}^{\prime}\right)\right] \\
& \times \delta\left(t-t^{\prime}\right)
\end{aligned}
$$

where $\langle\cdots\rangle$ represents the statistical average and $0 \leqslant \widetilde{\beta}^{-1}<1$ is the constant determined from the degree of coarse graining (see Ref. 41 for details). One can interpret that the temperature of the noise is the effective temperature $\widetilde{\beta}^{-1} T$, instead of the real temperature $T$. Also note that $\xi_{i}(\boldsymbol{r})$ can be generated easily by the algorithm proposed by van Vlimmeren and Fraaije. $^{42}$ It should be noted here that the free energy functional in Eq. (8) is generally some kind of effective free energy functional and is not equal to the free energy functional for the equilibrium state. ${ }^{41}$ In this work, the effective free energy functional is approximated as the equilibrium free energy functional [Eq. (6)]. This approximation corresponds to the assumption that all the polymer chains are fully relaxed, and thus this approximation neglects the viscoelasticity associated with the relaxation of polymer chains.

The dynamic equation [Eq. (8)] can be interpreted as the TDGL equation. In this case, the Onsager coefficient (or the mobility) $\phi_{i}(\boldsymbol{r}) / \zeta_{i}$ is proportional to $\phi_{i}(\boldsymbol{r})$. This is most important for the strong segregation region or in the situation that the solute concentration is sufficiently small. ${ }^{29,43,44}$ Here the TDGL equation with density dependent mobility and the free energy of ideal gases (the translational entropy of ideal gases) reduces to the diffusion equation (or the Smoluchowski equation). ${ }^{45}$ This suggests that the Flory-Huggins (or Bragg-Williams)-type free energy model should be used once the density dependent mobility is employed. Similarly, the density dependent mobility should be used if the FloryHuggins-type free energy model is employed. It is also noted that the TDGL equation with the constant mobility and the Flory-Huggins-type free energy leads to the unphysical singular behavior near $\phi_{i}(\boldsymbol{r})=0$.

For simplicity, all the segments are assumed to have the same friction coefficient $\left(\zeta_{i}=\zeta\right)$. Equation (8) can be rewritten by using $\phi_{i}(\boldsymbol{r})=\psi_{i}^{2}(\boldsymbol{r})$ as follows:

$$
\begin{aligned}
\frac{\partial \phi_{i}(\boldsymbol{r})}{\partial t} & =\nabla \cdot\left[\frac{k_{B} T}{\zeta} \psi_{i}^{2}(\boldsymbol{r}) \nabla\left[\frac{\delta\left(F / k_{B} T\right)}{\delta \psi_{i}(\boldsymbol{r})} \frac{\partial \psi_{i}(\boldsymbol{r})}{\partial \psi_{i}^{2}(\boldsymbol{r})}\right]\right]+\xi_{i}(\boldsymbol{r}, t) \\
& =\frac{k_{B} T}{2 \zeta}\left[\psi_{i}(\boldsymbol{r}) \nabla^{2} \mu_{i}(\boldsymbol{r})-\mu_{i}(\boldsymbol{r}) \nabla^{2} \psi_{i}(\boldsymbol{r})\right]+\xi_{i}(\boldsymbol{r}, t) \\
& =\frac{k_{B} T}{2 \zeta}\left[\psi_{i}(\boldsymbol{r}) \nabla^{2} \mu_{i}(\boldsymbol{r})-\mu_{i}(\boldsymbol{r}) \nabla^{2} \psi_{i}(\boldsymbol{r})+\frac{2 \zeta}{k_{B} T} \xi_{i}(\boldsymbol{r}, t)\right],
\end{aligned}
$$

where $\mu_{i}(\boldsymbol{r}) \equiv \delta\left(F / k_{B} T\right) / \delta \psi_{i}(\boldsymbol{r})$ is a kind of chemical potential field. It is noted that $\mu_{i}(\boldsymbol{r})$ is not singular at $\psi_{i}(\boldsymbol{r})=0$ and thus stable simulations can be performed [on the other hand, $\delta\left(F / k_{B} T\right) / \delta \phi_{i}(\boldsymbol{r})$ has a singularity and is numerically unstable].

By introducing rescaled variables defined as

$$
\tilde{t} \equiv \frac{k_{B} T}{2 \zeta} t,
$$

$$
\tilde{\xi}_{i}(\boldsymbol{r}, t) \equiv \frac{2 \zeta}{k_{B} T} \xi_{i}(\boldsymbol{r}, t),
$$

and substituting Eqs. (12) and (13) into Eqs. (11), (9), and (10), one obtains the dynamic equation for rescaled variables,

$$
\frac{\partial \phi_{i}(\boldsymbol{r})}{\partial \widetilde{t}}=\psi_{i}(\boldsymbol{r}) \nabla^{2} \mu_{i}(\boldsymbol{r})-\mu_{i}(\boldsymbol{r}) \nabla^{2} \psi_{i}(\boldsymbol{r})+\widetilde{\xi}_{i}(\boldsymbol{r}, \widetilde{t}),
$$

and the fluctuation dissipation relation for the rescaled noise field,

$$
\begin{aligned}
& \left\langle\widetilde{\xi}_{i}(\boldsymbol{r}, \widetilde{t})\right\rangle=0, \\
& \left\langle\widetilde{\xi}_{i}(\boldsymbol{r}, \widetilde{t}) \widetilde{\xi}_{j}\left(\boldsymbol{r}^{\prime}, \widetilde{t}\right)\right\rangle=-4 \widetilde{\beta}^{-1} \delta_{i j} \nabla \cdot\left[\phi_{i}(\boldsymbol{r}) \nabla \delta\left(\boldsymbol{r}-\boldsymbol{r}^{\prime}\right)\right] \delta(\widetilde{t}-\widetilde{t}) .
\end{aligned}
$$

It should be noted here that the magnitude of the noise $\widetilde{\xi}_{i}(\boldsymbol{r}, \widetilde{t})$ depends only on $\widetilde{\beta}^{-1}$. The temperature change corresponds to the change of the time scale (since $t \propto \tilde{t} / T$ ) and the change of the $\chi$ parameter. However, notice that to return from the rescaled time $\tilde{t}$ (which is used in the actual simulations) to the real time scale $t$, one has to multiply the factor $2 \zeta / k_{B} T$.

Note that one can set $k_{B} T / 2 \zeta=1$ instead of introducing rescaled variables $\left(\widetilde{t}\right.$ and $\left.\widetilde{\xi}_{i}\right)$. This corresponds to setting the effective diffusion coefficient for the monomer to unity (strictly speaking, the half of the effective diffusion coefficient is set to unity). This change can be done without losing generality, as shown above. To return to the real time scale, the factor $2 \zeta / k_{B} T$ must be multiplied to the rescaled time. This factor can be estimated from the experimental diffusion data. (The real time scale is estimated in Sec. IV B.)

It should be noted here that Eqs. (14) and (16) imply that the annealing process (the temperature change process) corresponds to the change of the $\chi$ parameter, and the magnitude of the noise is not changed because there are no other parameters related to $T$. The magnitude of the noise is characterized only by $\widetilde{\beta}^{-1}$ in the rescaled units.

\section{SIMULATION}

Equation (14) is solved numerically in three dimensions. The chemical potential $\mu_{i}(\boldsymbol{r})$ can be calculated from the free energy functional [Eq. (6)], whereas thermal noise $\xi_{i}(\boldsymbol{r}, t)$ is calculated by the van Vlimmeren and Fraaije's algorithm. ${ }^{42}$

\section{A. Numerical scheme}

Simulations are started from the homogeneous state $\left[\phi_{i}(\boldsymbol{r})=\bar{\phi}_{i}\right.$, where $\bar{\phi}_{i}$ is the spatial average of $\left.\phi_{i}(\boldsymbol{r})\right]$. Each step of the time evolution in the simulation is as follows.

(1) Calculate the $\psi$ field $\psi_{i}(\boldsymbol{r})$ and the chemical potential field $\mu_{i}(\boldsymbol{r})$ from the density field $\phi_{i}(\boldsymbol{r})$.

(2) Generate the thermal noise $\widetilde{\xi}_{i}(\boldsymbol{r}, \widetilde{t})$.

(3) Calculate the Lagrange multiplier $P(\boldsymbol{r})$.

(4) Evolve the density field $\phi_{i}(\boldsymbol{r})$ by time step $\Delta \widetilde{t}$, using $\psi_{i}(\boldsymbol{r}), \mu_{i}(\boldsymbol{r})$, and $\widetilde{\xi}_{i}(\boldsymbol{r}, \widetilde{t})$.

(5) Return to step (1). 
Here the numerical scheme for the simulations is described in detail. As mentioned above, the dynamic density functional equation [Eq. (8)] is reduced to the diffusion equation for the Flory-Huggins-type translational entropy functional. The Flory-Huggins translational entropy term is on the free energy [Eq. (6)]. Thus the dynamic equation [Eq. (14)] contains the standard diffusion term (Laplacian term).

$$
\frac{\partial \phi_{i}(\boldsymbol{r})}{\partial \tilde{t}}=2 \widetilde{C}_{i} \nabla^{2} \phi_{i}(\boldsymbol{r})+\cdots
$$

where $\widetilde{C}_{i}=f_{i} C_{i i}(i=A, B)$ and $\widetilde{C}_{s}=1$. The numerical stability of the diffusion-type equation can be improved by the implicit schemes. In the previous work ${ }^{18}$ the alternating direction implicit (ADI) scheme ${ }^{46}$ was employed for the Laplacian term. In this work the ADI scheme is employed to improve stability, as the numerical scheme for the equilibrium simulations.

Each time evolution step (time step $\Delta \widetilde{t}$ ) is split into three substeps (time step $\Delta \widetilde{t} / 3$ ). The update scheme for the density field from $\phi_{i}^{(n)}(\boldsymbol{r}) \equiv \phi_{i}(\boldsymbol{r}, \tilde{t}+n \Delta \widetilde{t} / 3)$ to $\phi_{i}^{(n+1)}(\boldsymbol{r})$ is as follows. (For simplicity, the continuum expression is used for the position $\boldsymbol{r}$ and the differential operators $\partial^{2} / \partial x^{2}, \partial^{2} / \partial y^{2}, \partial^{2} / \partial z^{2}$, and $\nabla^{2}$. In real simulations the standard lattice is used for the position and the center difference operators for the differential operators. ${ }^{46}$ )

$$
\begin{aligned}
{\left[1-\frac{2 \Delta \widetilde{t}}{3} \widetilde{C}_{i} \frac{\partial^{2}}{\partial x^{2}}\right] \phi_{i}^{(1)}(\boldsymbol{r})=} & \phi_{i}^{(0)}(\boldsymbol{r})+\frac{\Delta \widetilde{t}}{3}\left[-2 \widetilde{C}_{i} \frac{\partial^{2}}{\partial x^{2}} \phi_{i}^{(0)}(\boldsymbol{r})\right. \\
& +\psi_{i}^{(0)}(\boldsymbol{r}) \nabla^{2} \mu_{i}^{(0)}(\boldsymbol{r})-\mu_{i}^{(0)}(\boldsymbol{r}) \\
& \left.\times \nabla^{2} \psi_{i}^{(0)}(\boldsymbol{r})+\widetilde{\xi}_{i}^{(0)}(\boldsymbol{r}, \widetilde{t})\right], \\
{\left[1-\frac{2 \Delta \widetilde{t}}{3} \widetilde{C}_{i} \frac{\partial^{2}}{\partial y^{2}}\right] \phi_{i}^{(2)}(\boldsymbol{r})=} & \phi_{i}^{(1)}(\boldsymbol{r})+\frac{\Delta \widetilde{t}}{3}\left[-2 \widetilde{C}_{i} \frac{\partial^{2}}{\partial y^{2}} \phi_{i}^{(1)}(\boldsymbol{r})\right. \\
& +\psi_{i}^{(1)}(\boldsymbol{r}) \nabla^{2} \mu_{i}^{(1)}(\boldsymbol{r})-\mu_{i}^{(1)}(\boldsymbol{r}) \\
& \left.\times \nabla^{2} \psi_{i}^{(1)}(\boldsymbol{r})+\widetilde{\xi}_{i}^{(1)}(\boldsymbol{r}, \widetilde{t})\right], \\
\left.1-\frac{2 \Delta \widetilde{t}}{3} \widetilde{C}_{i} \frac{\partial^{2}}{\partial z^{2}}\right] \phi_{i}^{(3)}(\boldsymbol{r})= & \phi_{i}^{(2)}(\boldsymbol{r})+\frac{\Delta \widetilde{t}}{3}\left[-2 \widetilde{C}_{i} \frac{\partial^{2}}{\partial z^{2}} \phi_{i}^{(2)}(\boldsymbol{r})\right. \\
& +\psi_{i}^{(2)}(\boldsymbol{r}) \nabla^{2} \mu_{i}^{(2)}(\boldsymbol{r})-\mu_{i}^{(2)}(\boldsymbol{r}) \\
& \left.\times \nabla^{2} \psi_{i}^{(2)}(\boldsymbol{r})+\widetilde{\xi}_{i}^{(2)}(\boldsymbol{r}, \widetilde{t})\right] .
\end{aligned}
$$

In each step, the chemical potential $\mu_{i}^{(n)}(\boldsymbol{r})$ and $\xi_{i}^{(n)}(\boldsymbol{r})$ are regenerated. The numerical scheme for the calculation of the chemical potential $\mu_{i}^{(n)}(\boldsymbol{r})$ is found in Ref. 18. Equations (18)-(20) can be solved easily by using the numerical scheme for the cyclic tridiagonal matrix. ${ }^{46}$

$\psi_{i}^{(n)}(\boldsymbol{r})$ needs to be calculated in order to calculate the chemical potential $\mu_{i}^{(n)}(\boldsymbol{r})$. Because of the thermal noise and numerical error, the condition $0 \leqslant \phi_{i}^{(n)}(\boldsymbol{r}) \leqslant 1$ is not always satisfied. Thus $\psi_{i}^{(n)}(\boldsymbol{r})$ is written as

$$
\psi_{i}^{(n)}(\boldsymbol{r})= \begin{cases}0 & \left(\phi_{i}^{(n)}(\boldsymbol{r})<0\right) \\ 1 & \left(\phi_{i}^{(n)}(\boldsymbol{r})>1\right) \\ \sqrt{\phi_{i}^{(n)}(\boldsymbol{r})} & (\text { otherwise }) .\end{cases}
$$

This avoids numerical difficulty associated with the negative value of $\phi_{i}^{(n)}(\boldsymbol{r})$.

The noise field $\xi_{i}^{(n)}(\boldsymbol{r}, \widetilde{t})$ is calculated at each ADI time step, by using the scheme described in Appendix A (notice that the size of the time step here is $\Delta \widetilde{t} / 3$ instead of $\Delta \widetilde{t}$ ).

The Lagrange multiplier $P(\boldsymbol{r})$ is also updated at each ADI time step by the following scheme. Because of the large thermal noise, the incompressible condition is not always satisfied. Thus a roughly approximated and relatively simple scheme is used to calculate $P(\boldsymbol{r})$. If one ignores the terms in the chemical potential except for the Lagrange multiplier term, the ADI update scheme can be approximately written as

$$
\phi_{i}^{(n+1)}(\boldsymbol{r}) \approx \phi_{i}^{(n)}(\boldsymbol{r})+\frac{\Delta \widetilde{t}}{3} \nabla \cdot\left[\phi_{i}^{(n)}(\boldsymbol{r}) \nabla P(\boldsymbol{r})\right] .
$$

From Eq. (22)

$$
\begin{aligned}
\sum_{i} \phi_{i}^{(n+1)}(\boldsymbol{r}) \approx & \sum_{i} \phi_{i}^{(n)}(\boldsymbol{r})+\frac{\Delta \widetilde{t}}{3} \nabla \cdot\left[\sum_{i} \phi_{i}^{(n)}(\boldsymbol{r}) \nabla P(\boldsymbol{r})\right] \\
& \times \frac{1}{\Delta \widetilde{t} / 3}\left[\sum_{i} \phi_{i}^{(n+1)}(\boldsymbol{r})-\sum_{i} \phi_{i}^{(n)}(\boldsymbol{r})\right] \\
\approx & \nabla^{2} P(\boldsymbol{r})-\nabla \cdot\left[\left[1-\sum_{i} \phi_{i}^{(n)}(\boldsymbol{r})\right] \nabla P(\boldsymbol{r})\right] .
\end{aligned}
$$

Using the constraint $\sum_{i} \phi_{i}^{(n+1)}(\boldsymbol{r})=1$ and assuming $\mid 1-\Sigma_{i}$ $\times \phi^{(n)}(\boldsymbol{r}) \mid \ll 1$, finally

$$
\nabla^{2} P(\boldsymbol{r}) \approx \frac{1}{(\Delta \widetilde{t} / 3)}\left[1-\sum_{i} \phi_{i}^{(n)}(\boldsymbol{r})\right] .
$$

Equation (24) and the long range interaction terms [which contains the Green function $\left.\widetilde{\mathcal{G}}\left(\boldsymbol{r}-\boldsymbol{r}^{\prime}\right)\right]$ in the chemical potential $\mu_{i}(\boldsymbol{r})$ can be calculated by several numerical methods for partial differential equations. Here they are calculated by the direct method, using FFTw3. ${ }^{47}$

\section{B. Results}

Parameters for the diblock copolymer are as follows: polymerization index $N=10$, block ratio $f_{A}=1 / 3, f_{B}=2 / 3$, and the spatial average of the volume fraction $\bar{\phi}_{p}=0.2$. The cutoff length for the long range interaction should be set appropriately. If there are no solvent diblock copolymers form strongly segregated microphase separation structure (currently interests lies in the strong segregation region). Thus from Eq. (7) $\lambda \propto N^{2 / 3} b \simeq 4$.64. Here $\lambda$ is set to $\lambda=5$. The validity of this value is argued later (see also Appendix C).

Parameters for the solvent are as follows: the spatial average of the volume fraction $\bar{\phi}_{s}=1-\bar{\phi}_{p}=0.8$. Flory-Huggins $\chi$ parameters are $\chi_{A B}=2.5, \chi_{A S}=-0.5$, and $\chi_{B S}=5$ (the $A$ monomer is hydrophilic and the $B$ monomer is hydrophobic), and the Kuhn length is set to unity $(b=1)$. All the simulations 

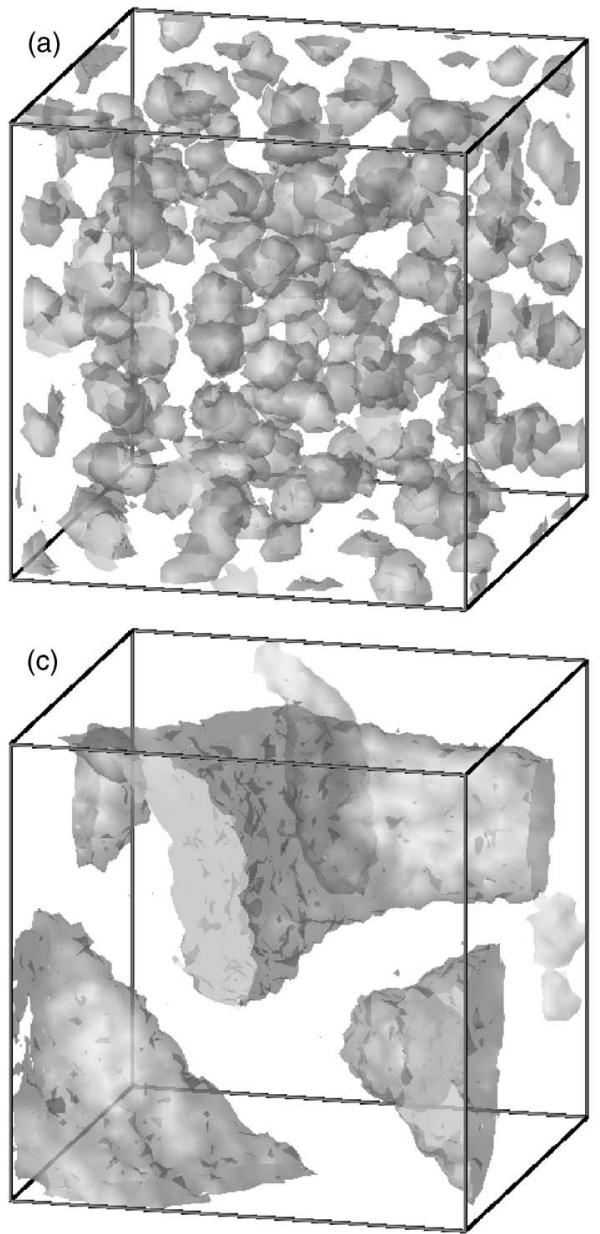

J. Chem. Phys. 126, $114902(2007)$
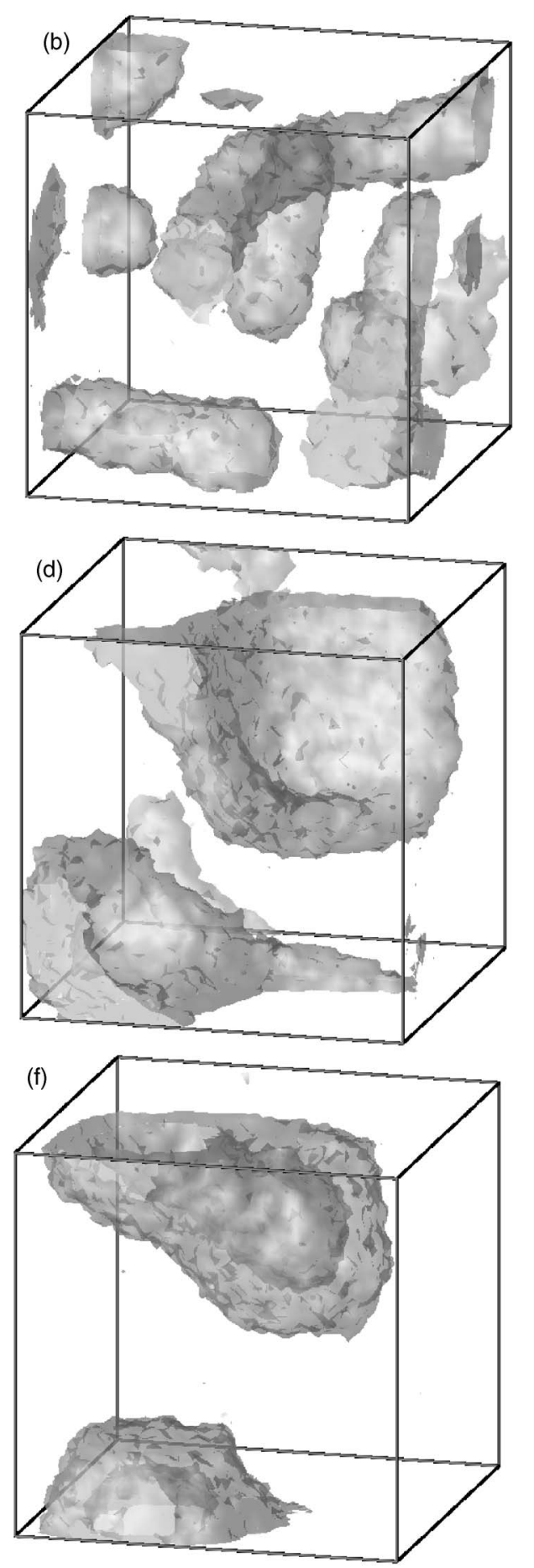

FIG. 2. Snapshots of the dynamics simulation for the amphiphilic diblock copolymer solution $\left(\bar{\phi}_{p}=0.2\right)$. Gray surface is the isodensity surface for the hydrophobic subchain, $\phi_{B}(\boldsymbol{r})=0.5$. (a) $\tilde{t}=6.25$, (b) $\tilde{t}=312.5$, (c) $\tilde{t}=1562.5$, (d) $\tilde{t}=3125$, (e) $\tilde{t}=4687.5$, and (f) $\tilde{t}$ $=6250$.

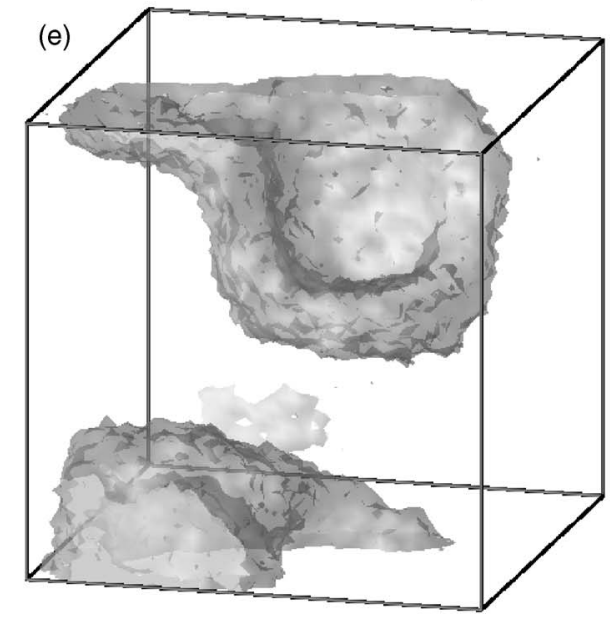

are carried out in three dimensions. The size of the simulation box is $24 b \times 24 b \times 24 b$ and the number of lattice points is $48 \times 48 \times 48$. The periodic boundary condition is applied. The time step is set to $\Delta \widetilde{t}=0.0025$ and the magnitude of noise is $\widetilde{\beta}^{-1}=0.0390625$. The simulation is carried out up to 2500000 time steps (from $\tilde{t}=0$ to $\tilde{t}=6250$ ) and it requires about 20 days on a $2.8 \mathrm{GHz}$ Xeon work station.

The snapshots of dynamics simulations are shown in Fig. 2. The observed vesicle formation process is different from mechanism II, which is observed in the previous thermal equilibrium simulations ${ }^{17,18}$ and EPD simulations. ${ }^{24}$ First, spherical micelles are formed [Fig. 2(a)], then the micelles aggregate and become large [Fig. 2(b)], and a disklike micelle [Fig. 2(c)] appears. The disklike micelle is closed [Figs. 2(d) and 2(e)] to form a vesicle [Fig. 2(f)]. This vesicle formation process agrees with mechanism I, which is observed in the particle simulations. ${ }^{3-5}$

To confirm that the vesicle formation is independent of the random seed, simulations are then performed with different random seeds. Four simulations are performed here, and vesicles are obtained in two simulations. Figures 3 and 4 show the snapshots of vesicle formation processes obtained for different random seeds (all other parameters are the same as the previous simulation). The vesicle formation processes in Figs. 3 and 4 again agree with mechanism I. While the 

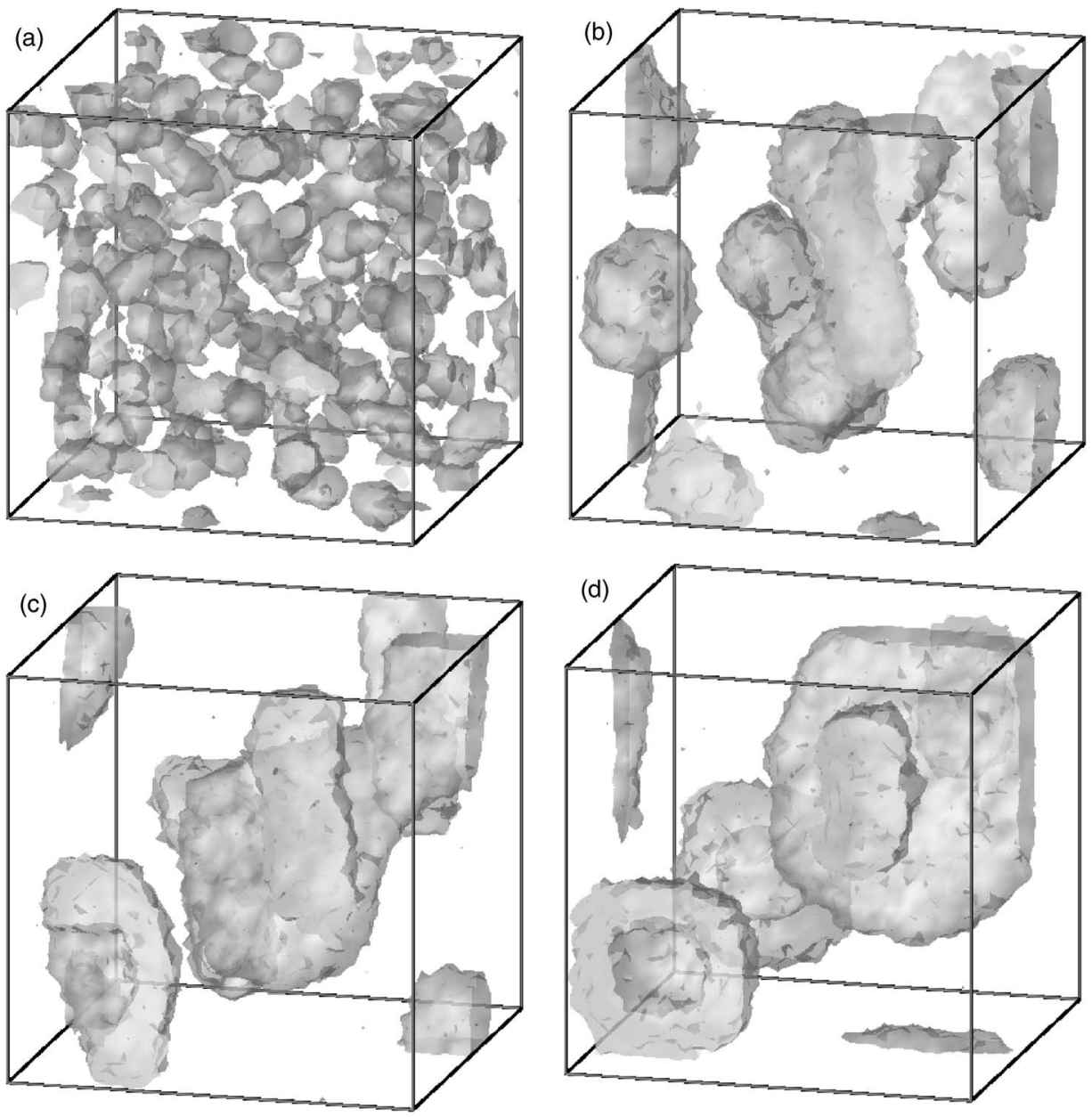

FIG. 3. Snapshots of the dynamics simulation for the amphiphilic diblock copolymer solution $\left(\bar{\phi}_{p}=0.2\right)$. Gray surface is the isodensity surface for the hydrophobic subchain, $\phi_{B}(\boldsymbol{r})=0.5$. (a) $\tilde{t}=6.25$, (b) $\tilde{t}=312.5$, (c) $\tilde{t}=1562.5$, and (d) $\tilde{t}=3125$.

vesicles are not observed for all the simulations, the vesicle formation process obtained by the previous simulation is not considered to be an artifact.

The characteristic size of the phase separation structure should be compared with the cutoff length $\lambda$. The characteristic size of the micellar structure can be found in the twodimensional cross section data or the density correlation function. Figures 5(a) and 5(b) show the two-dimensional cross section of the final structure $[\tilde{t}=6250$, Fig. 2(f) $]$. From Figs. 5(a) and 5(b) it is observed that the cutoff length $\lambda$ $=5$ is comparable to the characteristic size of the micelle bilayer structure. Figure 5(c) shows the radial averaged correlation function of the density field in the Fourier space $S_{A B}(q)$ defined as ${ }^{48}$

$$
S_{A B}(q) \equiv \frac{\sum_{q-\Delta / 2 \leqslant\left|\boldsymbol{q}^{\prime}\right|<q+\Delta / 2}\left[\phi_{A}\left(\boldsymbol{q}^{\prime}\right) / f_{A}-\phi_{B}\left(\boldsymbol{q}^{\prime}\right) / f_{B}\right]^{2}}{\sum_{q-\Delta / 2 \leqslant\left|\boldsymbol{q}^{\prime}\right|<q+\Delta / 2} 1},
$$

where $\phi_{i}(\boldsymbol{q})$ is the Fourier transform of the density field and $\Delta$ is the width of the shell in the Fourier space (here $\Delta$ $=0.5 \times 2 \pi)$. The correlation functions have peaks at $q / 2 \pi$ $\simeq 0.15$. Thus the characteristic wavelength for the corona (the $A$ subchain rich region) and the core (the $B$ subchain rich region) is $\simeq 6.7$. While the resolution (the shell width $\Delta$ ) is not fine, the characteristic size of the structure is comparable with the value of the cutoff length, $\lambda=5$. Therefore the value of $\lambda$ used in this simulation is considered to be appropriate and valid (see also Appendix C).

Figure 6 shows various micellar structures obtained from simulations for different volume fractions $\bar{\phi}_{p}=0.1,0.15$, 0.25 , and 0.3. Simulations are performed up to 1250000 time steps (from $\tilde{t}=0$ to $\tilde{t}=3125$ ). All other parameters are the same as those in Fig. 2. If the volume fraction of the polymer is small [Figs. 6(a) and 6(b)], only small micelles [spherical micelles in Fig. 6(a) and disklike micelles in Fig. 6(b)] are formed. On the other hand, if the volume fraction of the polymer is large [Figs. 6(c) and 6(d)], bilayer structures are formed. Vesicles in Fig. 2 are observed only for the intermediate volume fraction.

In experiments, the mixed solvent (mixture of organic solvents and water) is often used to control the morphology of the micellar structures of block copolymers. ${ }^{49-51}$ If one assumes that the mixed solvent is the mixture of common solvent $\left(\chi_{A S}\right.$ and $\chi_{B S}$ are sufficiently small or negative) and water, the volume fraction of water mainly affects the interaction between the hydrophobic subchain and the solvent. $\chi_{B S}$ is changed to mimic these experiments.

Figure 7 shows the results of simulations for various $\chi_{B S}$ $\left(\chi_{B S}=0.2,0.25,0.3\right.$, and 0.35). Spherical micelles are formed for low $\chi_{B S}$ and cylindrical micelles are formed for high $\chi_{B S}$. This agrees with the experimental results qualitatively. Shen and Eisenberg ${ }^{49,50}$ reported that by controlling solvent condition, one can control the morphology of the block copoly- 

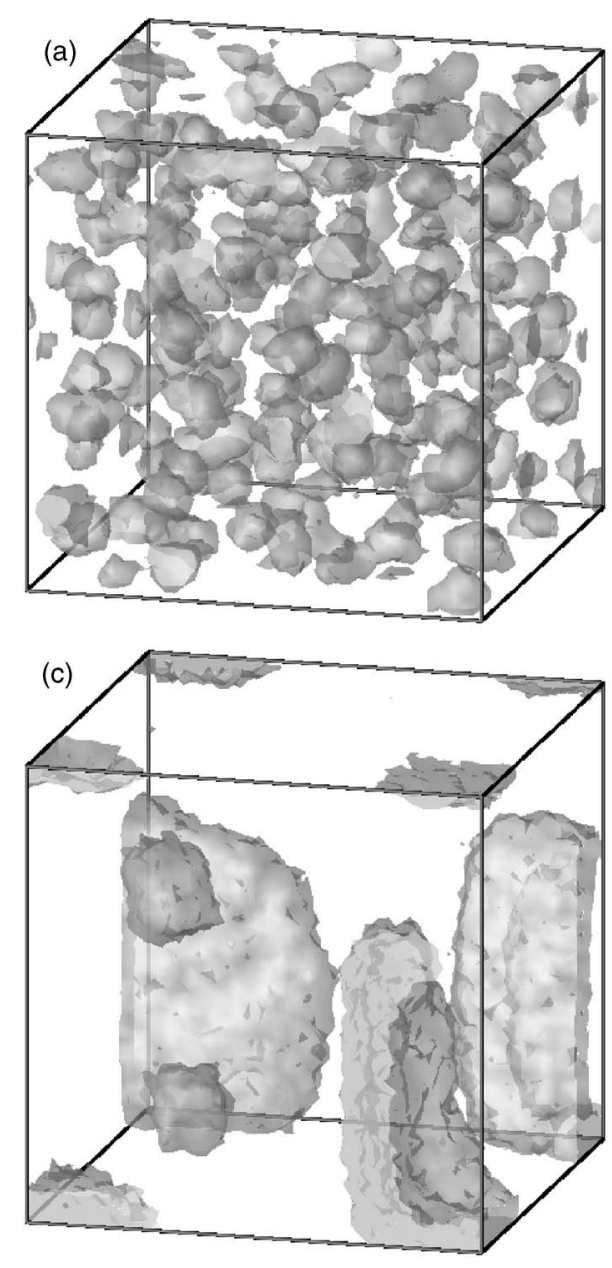
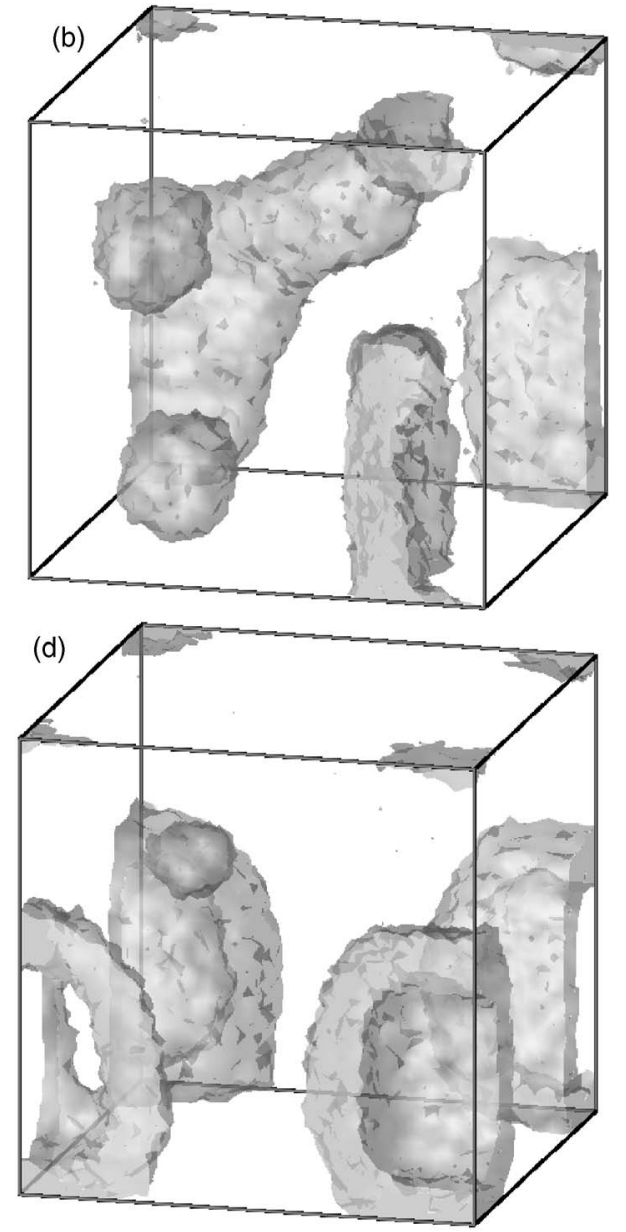

J. Chem. Phys. 126, 114902 (2007)
FIG. 4. Snapshots of the dynamics simulation for the amphiphilic diblock copolymer solution $\left(\bar{\phi}_{p}=0.2\right)$. Gray surface is the isodensity surface for the hydrophobic subchain, $\phi_{B}(\boldsymbol{r})=0.5$. (a) $\tilde{t}=6.25$, (b) $\tilde{t}=3125$, (c) $\tilde{t}=4687.5$, and (d) $\tilde{t}=6250$. (a)
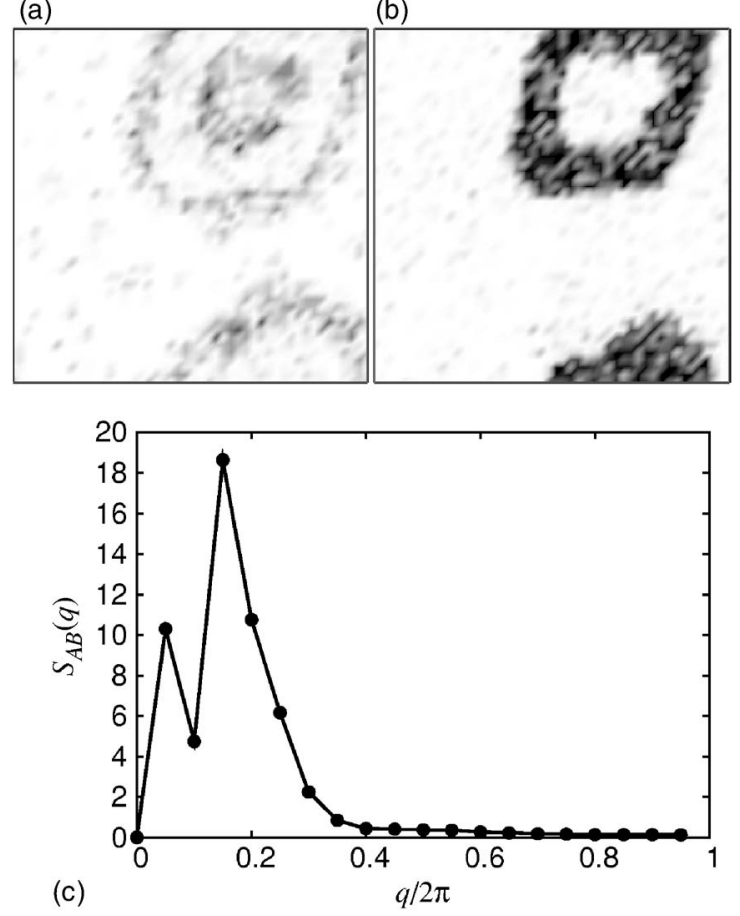

mer micelles. The control of the morphology is especially important for application use such as the drug delivery system. Here the solvent condition change is mimicked by changing the $\chi$ parameter between the hydrophobic segment and the solvent, $\chi_{B S}$. The $\chi$ parameter at $\widetilde{t}=6250$ [Fig. 2(f)] is changed from $\chi_{B S}=5$ to $\chi_{B S}=2.5$ or 3 , and the simulations are performed up to $\tilde{t}=9375$. The snapshots at $\tilde{t}=9375$ are shown in Fig. 8. The vesicle is changed into spherical micelles [Fig. 8(a)] or cylindrical micelles [Fig. 8(b)]. The resulting morphologies are consistent with the morphologies obtained by the simulations started from the homogeneous state [Figs. 7(b) and 7(c)].

\section{DISCUSSION}

From the dynamics simulations based on the density functional theory for amphiphilic diblock copolymers, one could observe the time evolution of spontaneous formation of vesicles. The micellar structures depending on the volume fraction of polymers were viewed. The observed vesicle formation process is the same as the results of the previous particle model simulations (mechanism I). At the initial stage of time evolution, rapid formation of small spherical micelles is observed. After the small micelles are formed, they aggregate each other by collision and become larger micelles. Finally, the large disklike micelles are closed and become

FIG. 5. Two dimensional cross sections of density field for (a) $\phi_{A}(\boldsymbol{r})$ and (b) $\phi_{B}(\boldsymbol{r})$. (c) The density correlation function $S_{A B}(\boldsymbol{q}) . \tilde{t}=6250$. 

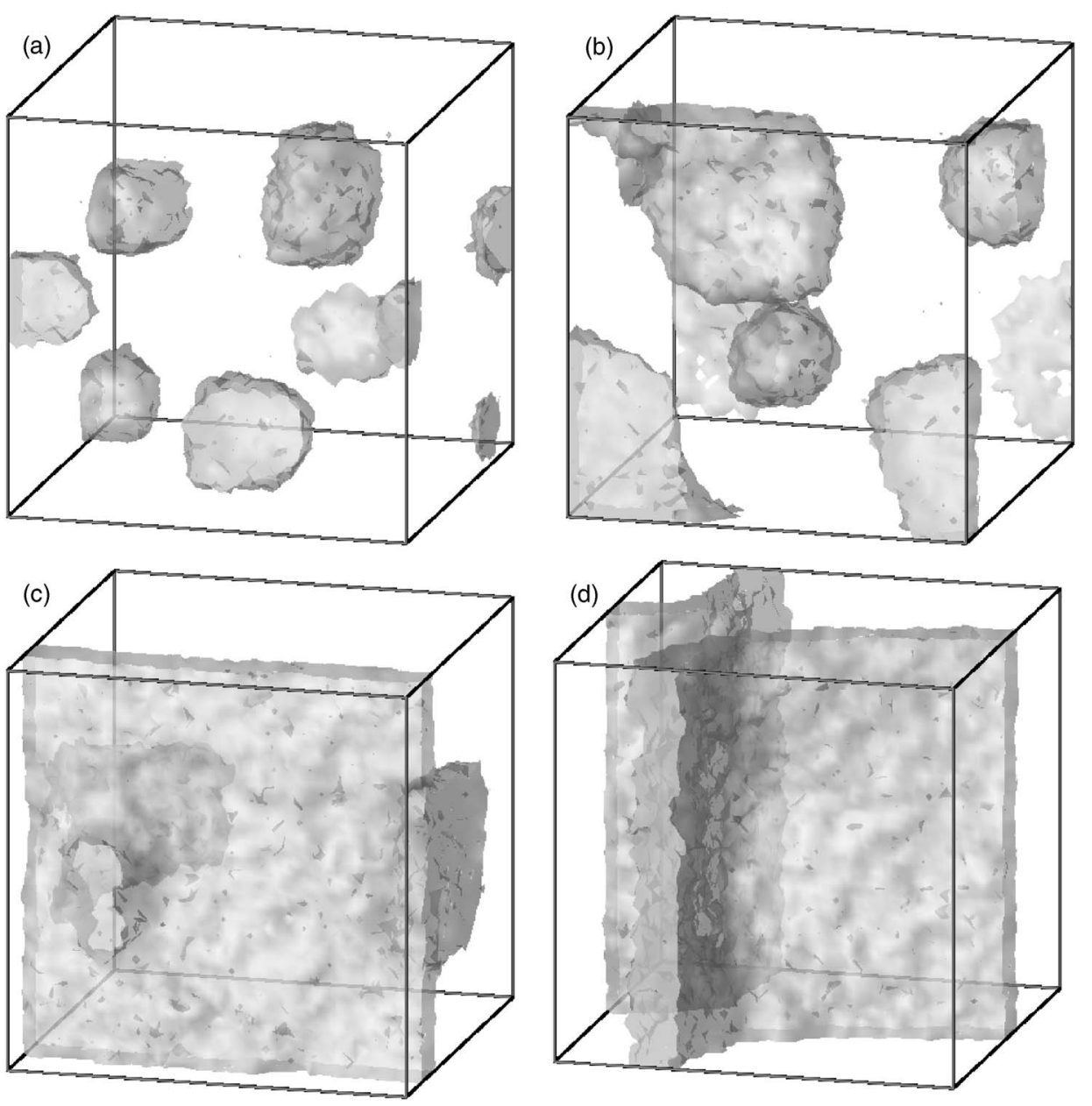

FIG. 6. Snapshots of the dynamics simulations for amphiphilic diblock copolymer solutions with various volume fractions. Gray surface is the isodensity surface for the hydrophobic subchain, $\phi_{B}(\boldsymbol{r})=0.5$ at $\tilde{t}=3125$. (a) $\bar{\phi}_{p}=0.1$, (b) $\bar{\phi}_{p}=0.15$, (c) $\bar{\phi}_{p}=0.25$, and (d) $\bar{\phi}_{p}=0.3$. $\left[\bar{\phi}_{p}=0.2\right.$ corresponds to Fig. 2(d).] vesicles. The late stage process, collision process and the close-up process, is slower than the initial stage, as mechanism I.

There are several important differences between this model and the standard TDGL model for the weak segregation limit. Some essential properties of this model will now be mentioned. First, our model can be applied for the strong segregation region, at least qualitatively. This is especially important, since the vesicles are observed in the strong segregation region.

Second, the interaction between the hydrophilic subchain and solvent should be handled carefully. If the hydrophilic interaction $\left(\chi_{A S}\right)$ is too small or both subchains are hydrophobic, the system undergoes macrophase separation and separates into block copolymer rich region and solvent rich region. In such a situation, vesicles are not formed and onion structures ${ }^{19-22}$ are formed instead.

Third, rather large thermal noise was applied to the system. Recently, Zhang and Wang ${ }^{52}$ have shown that the glass transition temperature and the spinodal line (stability limit of the disordered phase) for the microphase separation in diblock copolymer melts are quite close. This implies that microphase separated structures in the block copolymer systems are intrinsically glassy at the strong segregation region. Without sufficiently large and realistic thermal noise, the system is completely trapped at intermediate metastable structures (in most cases, spherical micelles) and thus thermodynamically stable structures (vesicles) will never be achieved.
As mentioned above, the magnitude of the noise is determined by the characteristic time scale and the factor $\widetilde{\beta}^{-1}$ is independent of the temperature. Thus the noise is important for mesoscopic systems even if the temperature of the system is not high.

\section{A. Thermal noise}

Here, thermal noise is discussed in detail. Intuitively, the large thermal noise is needed to overcome the free energy barrier. In the kinetic pathway of usual macrophase separation processes (for example, phase separations in homopolymer blends), there are not so large free energy barriers. Therefore, in most cases, the simulations for such systems work well with small thermal noise or without thermal noise. On the other hand, in the kinetic pathway of vesicle/micelle formation processes, there are various large free energy barriers (for example, in the collision and coalescence process of spherical micelles). However, it is not clear and established how to determine the magnitude of the thermal noise for such systems.

As mentioned, the thermal noise in Eq. (8) is expressed by using the effective temperature $\widetilde{\beta}^{-1} T$, instead of the real temperature $T$, and $\widetilde{\beta}^{-1}$ is determined from the degree of coarse graining. Intuitively, this means that the coarse graining procedure decreases the degree of the freedom of the field. The similar approach can be found in the van Vlimmeren and Fraaije theory. ${ }^{42}$ They employed the noise scaling 

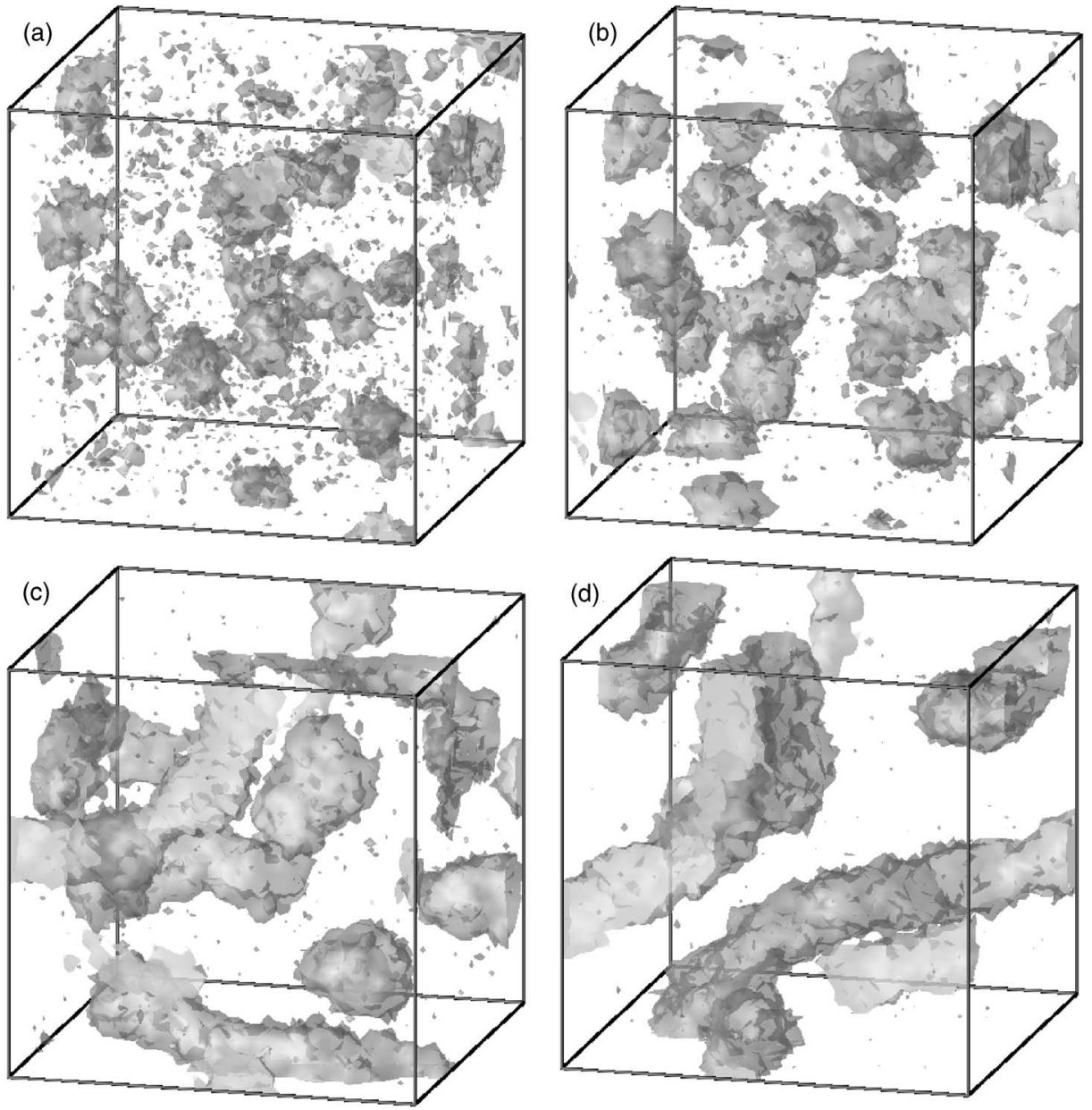

J. Chem. Phys. 126, 114902 (2007)

FIG. 7. Snapshots of the dynamics simulations for amphiphilic diblock copolymer solutions with various $\chi_{B S}$ (the $\chi$ parameter between the hydrophobic chain and the solvent). Gray surface is the isodensity surface for the hydrophobic subchain, $\phi_{B}(\boldsymbol{r})=0.5$ at $\tilde{t}$ $=3125$. (a) $\chi_{B S}=2$, (b) $\chi_{B S}=2.5$, (c) $\chi_{B S}=3$, and (d) $\chi_{B S}=3.5$. $\left[\chi_{B S}=5\right.$ corresponds to Fig. 2(d).] parameter $\Omega$ to express the effective degree of freedom in the simulation cell. These two approaches are similar, and, in fact, the relation between them can be written.

$$
\Omega=\frac{\left|\boldsymbol{h}_{x}\right|\left|\boldsymbol{h}_{y}\right|\left|\boldsymbol{h}_{z}\right|}{\widetilde{\beta}^{-1}}
$$

where $\boldsymbol{h}_{\alpha}$ is the lattice vector. From Eq. (26) one can calculate the noise scaling parameter for this simulation. Using the parameters used in Sec. III B, $\Omega=\left(0.5^{3}\right) / 0.0390625=3.2$. It should be noticed that this value of the noise scaling parameter is much smaller than the values used in most of previous works $(\Omega=100)$ by Fraaije and co-workers. ${ }^{53-55}$ However, since the thermal noise plays an important role in the vesicle formation process, $\widetilde{\beta}^{-1}$ cannot be underestimated . One may consider that the mean field approximation and the free energy functional [Eq. (6)] does not hold for low noise scaling parameter systems. It may be true, but as shown by Dean, ${ }^{39}$ the dynamic density functional equation holds even if there are not large number of particles. Thus, here it is believed that the mean field approximation still holds for these systems.
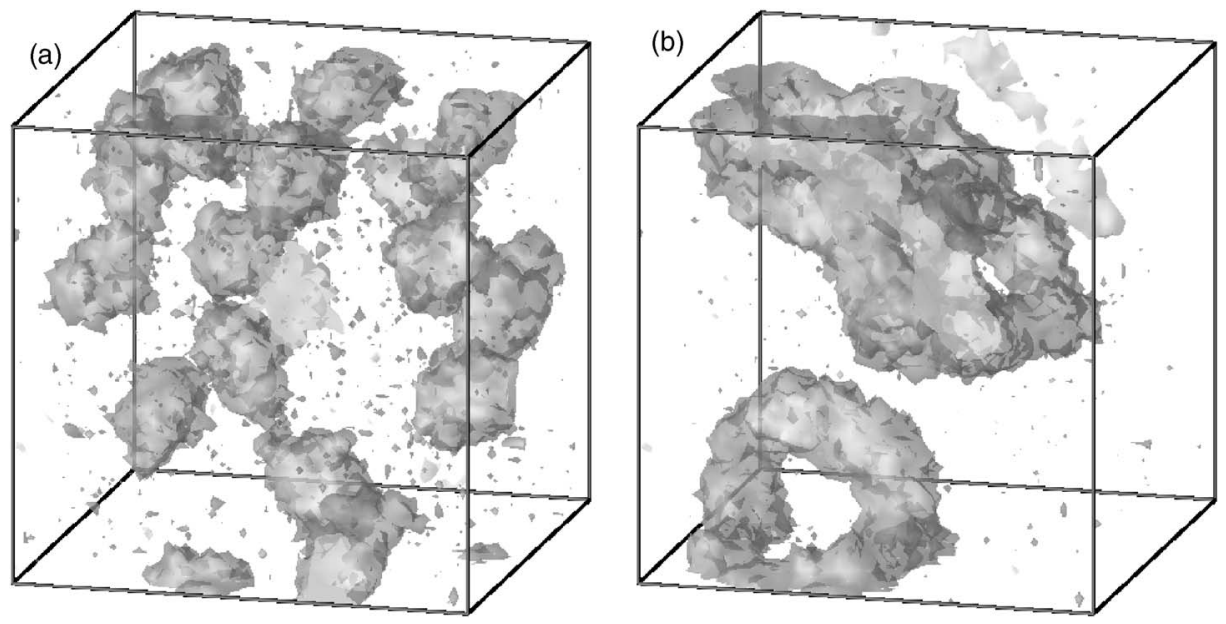

FIG. 8. Snapshots of the structural transition dynamics simulations. The $\chi$ parameter $\chi_{B S}$ is initially set to $\chi_{B S}$ $=5$ and at $\tilde{t}=6250, \chi_{B S}$ is set to lower value. Gray surface is the isodensity surface for the hydrophobic subchain, $\phi_{B}(\boldsymbol{r})=0.5$ at $\tilde{t}=9375$. (a) $\chi_{B S}=5$ $\rightarrow 2.5$ and (b) $\chi_{B S}=5 \rightarrow 3$. 

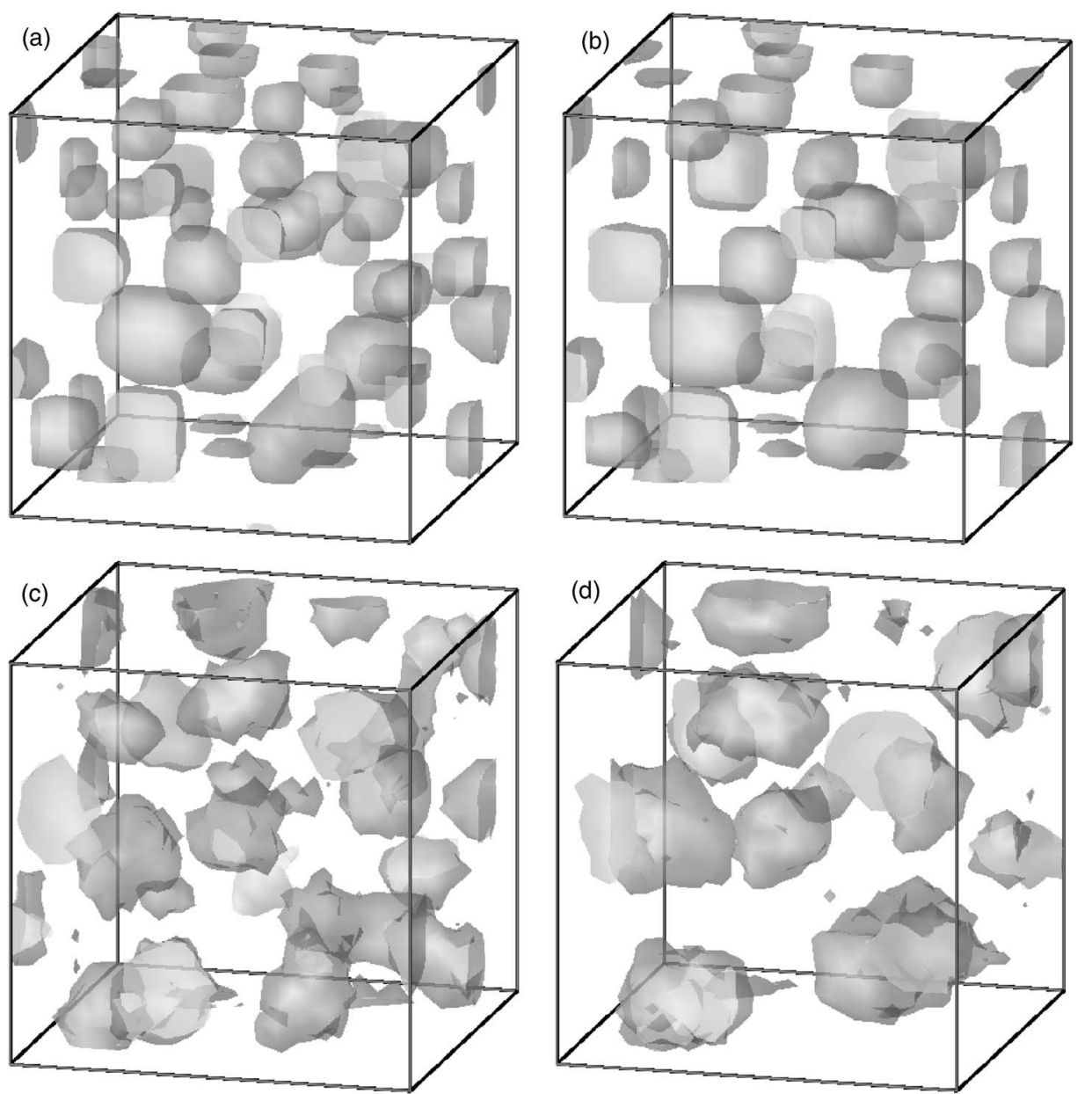

J. Chem. Phys. 126, 114902 (2007)

FIG. 9. Snapshots of the dynamics simulations for values of $\tilde{\beta}^{-1}$ at $\tilde{t}$ $=6.25,12.5$. $\quad[(\mathrm{a})$ and $\quad$ (b) $] \quad \tilde{\beta}^{-1}$ $=0.00390625, \tilde{t}=6.25,12.5$; [(c) and (d)] $\tilde{\beta}^{-1}=0.0390625, \quad \tilde{t}=6.25,12.5$; and $[(\mathrm{e})$ and (f) $] \widetilde{\beta}^{-1}=0.390625, \tilde{t}$ $=6.25,12.5$.


To show the necessity of the large thermal noise in the simulations, several simulations are performed with various values of $\widetilde{\beta}^{-1}$. It is expected that the simulations for small boxes are sufficient to see the effect of the magnitude of the thermal noise, since the effect is large even for the early stage of the dynamics. Thus, simulations for small systems $(12 b \times 12 b \times 12 b$ system size, $24 \times 24 \times 24$ lattice points $)$ are performed with different values of $\widetilde{\beta}^{-1} ; \quad \tilde{\beta}^{-1}$ $=0.00390625,0.0390625,0.390625$. All the other parameters are set to the previous vesicle formation simulations in Sec. III B (Fig. 2). Figure 9 shows the snapshots of the simulations. For the small thermal noise case [Figs. 9(a) and 9(b)], many micelles with sharp interfaces can be observed. For the large thermal noise case used in Sec. III B [Figs. 9(c) and 9(d)], One can observe the collision-coalescence type coarsening process of micelles, driven by the thermal noise. For the larger thermal noise case [Figs. 9(e) and 9(f)] no structures are observed. (It is thought this is due to the thermal noise being too large.) Thus, it can be concluded that the large (but not too large) thermal noise is required. This result is consistent with the proposition of van Vlimmeren et al. ${ }^{56}$ They carried out two dimensional simulations for dense amphiphilic triblock copolymer solutions, with the full (large) noise and the reduced (small) noise, and proposed that the 
real systems should have some intermediate noise (smaller than the full noise, but larger than the reduced noise).

It should be noted that simulations with small thermal noise are very difficult to perform for systems, because they are in the strong segregation region. The simulation with the small noise shows strong lattice anisotropy. This means that there are very sharp interfaces which often leads to numerical instability, and thus finer lattices should be used for such systems. On the other hand, such strong lattice anisotropy is not observed in the simulations with large noise. It is believed that the large thermal noise stirs the density fields and stabilizes the simulation.

At the end of this section, it is noted that it is difficult to determine the parameter $\widetilde{\beta}^{-1}$ theoretically. The estimation of the magnitude of the thermal noise is the open problem.

\section{B. Comparison with experiments}

Next, the relation between the simulation results and the experiments is discussed. Many parameters were used for the simulations and they should be related to the real experimental parameters. Unfortunately, because the accuracy of this theory is not high and many approximations are involved, it is difficult to calculate the real parameters from the simulation parameters.

The most important parameters are the Flory-Huggins $\chi$ parameters. The $\chi$ parameters are experimentally measured or calculated from the solubility parameters. The $\chi$ parameter between the hydrophilic subchain and the hydrophobic subchain is in most cases sufficiently large to cause the microphase separation. For example, Bhargava et al. ${ }^{51}$ calculated the $\chi$ parameter between polystyrene (PS), which can be used as the hydrophobic monomer, ${ }^{2}$ and water as $\chi_{\text {PS, water }}=6.27$. Dormidontova ${ }^{57}$ measured the $\chi$ parameter between poly(ethylene oxide) (PEO) and water and reported that the $\chi$ parameter can be expressed as $\chi_{\mathrm{PEO} \text {,water }}$ $=-0.0615+70 / T$ ( $T$ is the absolute temperature $)$. Lam and Goldbeck-Wood $^{58}$ also measured $\chi_{\mathrm{PEO} \text {,water }}$ and obtained $\chi_{\text {PEO, water }}=1.35$. Xu et al. ${ }^{59}$ measured the interaction between $\mathrm{PS}$ and PEO and reported $\chi_{\mathrm{PS}, \mathrm{PEO}}=0.02-0.03$. Zhu et al. ${ }^{60}$ also measured $\chi_{\mathrm{PS}, \mathrm{PEO}}$ and obtained $\chi_{\mathrm{PS}, \mathrm{PEO}}=-0.00705$ $+21.3 / T$. The polymerization index of PS-PEO diblock copolymer used in the experiments ${ }^{51}$ is typically $\simeq 1000$, thus we expect that $\chi_{\mathrm{PS}, \mathrm{PEO}} N$ is sufficiently larger than the critical value $\chi_{c} N=10.495 .{ }^{14}$ Note that the polyelectrolytes such as poly(acrylic acid) is often used as the hydrophilic subchian. ${ }^{49,50}$ Such polyelectrolytes can be dissolved into water easily, and thus it is expected that the $\chi$ parameter between the polyelectrolytes and the water is negative.

Thus, it can be said that the diblock copolymers are in the strong segregation region, and the effect of water addition is expected to be large for hydrophobic subchains, but not so large for hydrophilic subchains. Thus consider that the simulation is qualitatively consistent with these experiments. Note, however, the $\chi$ parameter used in the simulations and measured by experiments cannot be compared directly because the value of the $\chi$ parameter depends on the definition of the segment, and the free energy functional [Eq. (6)] is not quantitatively as accurate as the SCF theory. ${ }^{22}$ Also note that most experiments are carried out in the very strong segregation region in which it is quite hard to perform continuum field simulations. While it is difficult to compare such simulations with real experiments, these simulations are expected to give qualitatively correct physical process since they take the crucial physical properties correctly; the hydrophobic interaction $\chi_{B S}$ is sufficiently large and the hydrophilic interaction $\chi_{A S}$ is small. These properties are not well considered in most of the previous dynamics simulations.

Other interesting properties are the size of the vesicles, the content of solvents inside vesicles, and the higher order structures. These properties cannot be discussed from the current results, since the system size is not large. There is only one vesicle in the simulation box (Figs. 2-4) and there may be the finite size effect (see also Appendix B). Nevertheless, it is believed that this model and simulation results are still valuable. These properties of vesicles should be observed if the simulations for large systems even though performing large scale simulations by the current models, algorithms, and CPU power is difficult.

The characteristic time scales are also interesting and important property. As mentioned above, the real time scale can be estimated from the diffusion data. Here, the time scale is estimated by using the empirical equation for diffusion coefficient of short polystyrene melts (at $T_{g}+125^{\circ} \mathrm{C}$ ) by Watanabe and Kotaka. ${ }^{61}$

$$
D \simeq 6.3 \times 10^{-5} M^{-1} \mathrm{~cm}^{2} / \mathrm{s},
$$

where $M$ is the molecular weight.

The number of monomers in one segment used in these simulations must be determined. Since the polymerization index of typical amphiphilic block copolymers used in experiments $^{50,51}$ is about $M \simeq 50-1000$, it is noted that each segment used in simulations contains about 10 monomer units. In these simulations, dimensionless segment size ( $b$ $=1$ ) was used. Thus, the size of the segment is also needed. The segment size of the styrene monomer is about $7 \AA$ (it can be calculated from the experimental data of the radius of gyration $\left.^{62,63}\right)$. So, if the Gaussian statistics for monomers in the segment are assumed, the size of the segment can be expressed as $b_{0} \simeq \sqrt{10} \times 7 \AA$.

The characteristic time scale for the system can now be estimated. If it is assumed that the polymer chains are not entangled and obey the Rouse dynamics, the characteristic time scale $\tau$ can be calculated as follows:

$$
\tau=\frac{2 \zeta b_{0}^{2}}{k_{B} T}=\frac{2 b_{0}^{2}}{N D}
$$

where $b_{0}$ is the size of the segment, $N$ is the number of segments in one polymer chain (polymerization index), and $D$ is the diffusion coefficient. $N$ and $M$ can be related as $M=104 \times 10 \times N$ and thus

$$
\tau \simeq \frac{2 \times 1040 \times\left(\sqrt{10} \times 7 \times 10^{-10} \mathrm{~m}\right)^{2}}{6.5 \times 10^{-9} \mathrm{~m}^{2} / \mathrm{s}} \simeq 1.6 \times 10^{-6} \mathrm{~s} .
$$

Thus it is known that the characteristic time scale in these simulations is about $1 \mu \mathrm{s}$, and the time scale of the vesicle 
formation is about $10 \mathrm{~ms}$. Of course the time scale estimated here is quite rough and cannot be compared with real experiments directly, but the time scale of this simulation is considered to be much smaller than the one of other experiments. The possible reasons are that the accuracy of the dynamic equation used for the simulation is not so good and that the sizes of the formed vesicles are small since the system size is small.

\section{Comparison with the EPD simulations}

Here, the present simulation results will be compared with EPD simulation results and the reason why the EPD simulations $^{24}$ and the DF simulations give qualitatively different results (mechanism II and mechanism I) will be discussed.

The main differences between the EPD simulations and our DF simulations are the free energy functional model and the dynamic equation. The EPD simulations use the free energy calculated from the SCF theory, while the DF simulations use the free energy functional [Eq. (6)]. It is known that the DF theory is less accurate than the SCF theory but gives qualitatively acceptable results. ${ }^{18,22}$ Thus it is difficult to consider that the accuracy of the free energy model affects the observed mechanisms. It is expected that mechanism I is reproduced if the free energy functional model is changed from Eq. (6) to more accurate SCF model.

The other difference is the dynamic equation. The EPD model employs nonlocal mobility model, but in the derivation of the dynamic equation, rather rough approximations are involved. ${ }^{64}$ Because of these approximations, the local mass conservation is not satisfied in the EPD simulations (unless the system is homogeneous). This may affect the dynamic behavior seriously. The mobility in the dynamic equation [Eq. (8)] is the local type, and thus it is considered to be less accurate than the nonlocal model. But in dynamic equation, local mass conservation is satisfied exactly. Thus, it is considered that the EPD simulations reproduce mechanism II because it does not satisfy the local mass conservation. It is expected that if the dynamic equation which satisfies the local mass conservation is used with nonlocal mobility and the SCF free energy, mechanism I should be reproduced.

\section{CONCLUSION}

It has been shown that the vesicle formation mechanism I can be reproduced by the simulation using the dynamic density functional equation [Eq. (8)] and the free energy functional [Eq. (6)]. The simulation results are qualitatively in agreement with other simulations. ${ }^{3,4}$ This is the first realistic vesicle formation dynamic simulation based on the field theoretical model. To reproduce mechanism I, one needs the free energy functional which can be applied to strong segregation regions, the large thermal noise, and the proper interaction parameters for hydrophilic and hydrophobic interactions. These conditions are not satisfied previous field theoretical dynamic simulations. It has also shown that the formation dynamics of various micellar structures (spherical micelles, cylindrical micelles, vesicles, and bilayers) and morphological transitions can be simulated by the model.
The morphological transitions can be reproduced only by changing the $\chi$ parameter which corresponds to the change of the solvent condition.

The merit of using the field theoretical approach is that one can use the Flory-Huggins interaction parameters, instead of potentials between segments which is required for particle simulations. It has been shown that one can mimic the solvent condition control by changing the $\chi$ parameter between the hydrophobic segment and the solvent and have shown that the solvent condition control actually causes morphological transitions. For coarse grained particle simulations, the microscopic potential cannot be used directly, and in most cases the phenomenological potential models are used. Of course for some particle simulations, such as the DPD, one can use the $\chi$ parameters by mapping them onto the DPD potential parameters. But such a method is based on the parameter fitting ${ }^{65}$ and not always justified. Thus it is considered that especially for many component systems, such as solutions of amphiphiles, the field theoretical approaches have advantages.

Although the $\chi$ parameters in the current model cannot be compared with the experiments directly, this data may help in understanding the experimental data or the physical mechanism. It will be possible to use the experimentally determined $\chi$ parameters by employing more accurate free energy model such as the SCF model ${ }^{15,16,27}$ and efficient numerical algorithms. (Note that recently Honda and Kawakatsu $^{66}$ proposed the hybrid theory of the DF and the SCF and reported that accurate and numerically efficient simulations can be performed by the hybrid method. By employing their method, accurate and fast simulations for the dynamics of micelles and vesicles may be possible.) The quantitative simulations will be for future work.

While the efficiency of this simulation method based on the density functional theory [Eqs. (6) and (14)] is larger than the SCF simulations, currently, it is still comparable to particle simulations. Besides, because the late stage of mechanism I is a very slow process, the current model still needs considerable computational costs. To study the late stage dynamics or large scale systems (for example, many vesicle systems), more coarse grained and numerically more efficient computational methods will be needed. Current work can be the basis of the further coarse grained field theoretical model.

\section{ACKNOWLEDGMENTS}

The author thanks T. Ohta for reading the manuscript and giving useful suggestions, and for helpful comments and discussions. Thanks are also due to H. Morita, T. Taniguchi, Y. Masubuchi, H. Frusawa, and T. Kawakatsu for helpful comments and discussions. This work is supported by the Research Fellowships of the Japan Society for the Promotion of Science for Young Scientists. 



J. Chem. Phys. 126, 114902 (2007)

FIG. 10. Snapshots of the dynamics simulations for amphiphilic diblock copolymer solutions with various small box sizes at $\tilde{t}=3125$. (a) box size of $6 b \times 6 b \times 6 b(12 \times 12 \times 12$ lattice points), (b) box size of $8 b \times 8 b \times 8 b$ $(16 \times 16 \times 16$ lattice points), (c) box size of $12 b \times 12 b \times 12 b(24 \times 24 \times 24$ lattice points), and (d) box size of $16 b \times 16 b \times 16 b(32 \times 32 \times 32$ lattice points). Supercells (size of $24 b \times 24 b$ $\times 24 b$ ) are shown. Small gray boxes show the real simulation box.

\section{APPENDIX A: NOISE GENERATION SCHEME}

In this section, the numerical scheme for generating random noise field is derived which satisfies the fluctuation dissipation relation [Eqs. (15) and (16)]. Such a noise field can be expressed as ${ }^{42}$

$$
\begin{aligned}
\widetilde{\xi}_{i}(\boldsymbol{r}, \widetilde{t}) & =\sqrt{4 \widetilde{\beta}^{-1}} \nabla \cdot\left[\sqrt{\phi_{i}(\boldsymbol{r})} \boldsymbol{\omega}_{i}(\boldsymbol{r}, \widetilde{t})\right] \\
& =2 \sqrt{\widetilde{\beta}^{-1}} \nabla \cdot\left[\psi_{i}(\boldsymbol{r}) \boldsymbol{\omega}_{i}(\boldsymbol{r}, \widetilde{t})\right],
\end{aligned}
$$

where $\boldsymbol{\omega}_{i}(\boldsymbol{r}, \widetilde{t})$ is the Gaussian white noise (vector) field which satisfies

$$
\begin{aligned}
& \left\langle\boldsymbol{\omega}_{i}(\boldsymbol{r}, \widetilde{t})\right\rangle=0, \\
& \left\langle\boldsymbol{\omega}_{i}(\boldsymbol{r}, \widetilde{t}) \boldsymbol{\omega}_{j}\left(\boldsymbol{r}^{\prime}, \widetilde{t}^{\prime}\right)\right\rangle=\delta_{i j} \delta\left(\boldsymbol{r}-\boldsymbol{r}^{\prime}\right) \delta\left(\widetilde{t}-\widetilde{t}^{\prime}\right) \mathbf{1},
\end{aligned}
$$

where $\mathbf{1}$ is the unit tensor.

To generate the noise numerically, the discretized version of Eq. (A1) is needed. The discretized noise field $\widetilde{\xi}_{i}(\boldsymbol{r}, \widetilde{t})$ is defined only on the lattice points. Here, the position of the lattice point is expressed as $\boldsymbol{r}=n_{x} \boldsymbol{h}_{x}+n_{y} \boldsymbol{h}_{y}+n_{z} \boldsymbol{h}_{z}$, where $\boldsymbol{h}_{\alpha}$ $(\alpha=x, y, z)$ is the lattice vector and $n_{\alpha}$ is the integer. The rescaled time $\tilde{t}$ is also discretized as $\tilde{t}=n_{t} \Delta \widetilde{t}$, where $n_{t}$ is the integer. The Laplacian operator is replaced by the standard centre finite difference operator as follows:

$$
\nabla^{2} f(\boldsymbol{r}) \rightarrow \sum_{\alpha=x, y, z} \frac{1}{\left|\boldsymbol{h}_{\alpha}\right|^{2}}\left[f\left(\boldsymbol{r}+\boldsymbol{h}_{\alpha}\right)-f(\boldsymbol{r})+f\left(\mathbf{r}-\boldsymbol{h}_{\alpha}\right)\right],
$$

where $f(\mathbf{r})$ is a discretized field defined on the lattice points. The delta functions are replaced as follows:

$$
\begin{aligned}
& \delta\left(\boldsymbol{r}-\boldsymbol{r}^{\prime}\right) \rightarrow \frac{\delta_{r r^{\prime}}}{\left|\boldsymbol{h}_{x}\right|\left|\boldsymbol{h}_{y}\right|\left|\boldsymbol{h}_{z}\right|}, \\
& \delta\left(t-t^{\prime}\right) \rightarrow \frac{\delta_{t t^{\prime}}}{\Delta \widetilde{t}},
\end{aligned}
$$

where $\delta_{r r^{\prime}}=\delta_{n_{x^{\prime}} n_{x}^{\prime}} \delta_{n_{y} n_{y}^{\prime}} \delta_{n_{z} n_{z}^{\prime}}$ and $\delta_{\tilde{t} t^{\prime}}=\delta_{n_{t} n_{t}^{\prime}}$.

The fluctuation dissipation relation for the second order moment [Eq. (16)] can be rewritten as

$$
\begin{aligned}
\left\langle\widetilde{\xi}_{i}(\boldsymbol{r}, \widetilde{t}) \widetilde{\xi}_{j}\left(\boldsymbol{r}^{\prime}, \widetilde{t}^{\prime}\right)\right\rangle= & -4 \widetilde{\beta}^{-1} \delta_{i j} \nabla \cdot\left[\psi_{i}^{2}(\boldsymbol{r}) \nabla \delta\left(\boldsymbol{r}-\boldsymbol{r}^{\prime}\right)\right] \delta\left(\tilde{t}-\widetilde{t}^{\prime}\right) \\
= & -4 \widetilde{\beta}^{-1} \delta_{i j} \nabla \cdot\left[\psi_{i}^{2}(\boldsymbol{r}) \nabla \frac{\psi_{i}(r) \delta\left(r-r^{\prime}\right)}{\psi_{i}(\boldsymbol{r})}\right] \\
& \times \delta\left(\widetilde{t}-\widetilde{t}^{\prime}\right) \\
= & -4 \widetilde{\beta}^{-1} \delta_{i j}\left[\psi_{i}(\boldsymbol{r}) \nabla^{2}\left[\psi_{i}(\boldsymbol{r}) \delta\left(\boldsymbol{r}-\boldsymbol{r}^{\prime}\right)\right]\right. \\
& \left.-\left[\psi_{i}(\boldsymbol{r}) \delta\left(\boldsymbol{r}-\boldsymbol{r}^{\prime}\right)\right] \nabla^{2} \psi_{i}(\boldsymbol{r})\right] \delta\left(\tilde{t}-\widetilde{t}^{\prime}\right) .
\end{aligned}
$$

Using Eqs. (A4)-(A6), the discretized version of the fluctuation dissipation relation (A7) is obtained. 

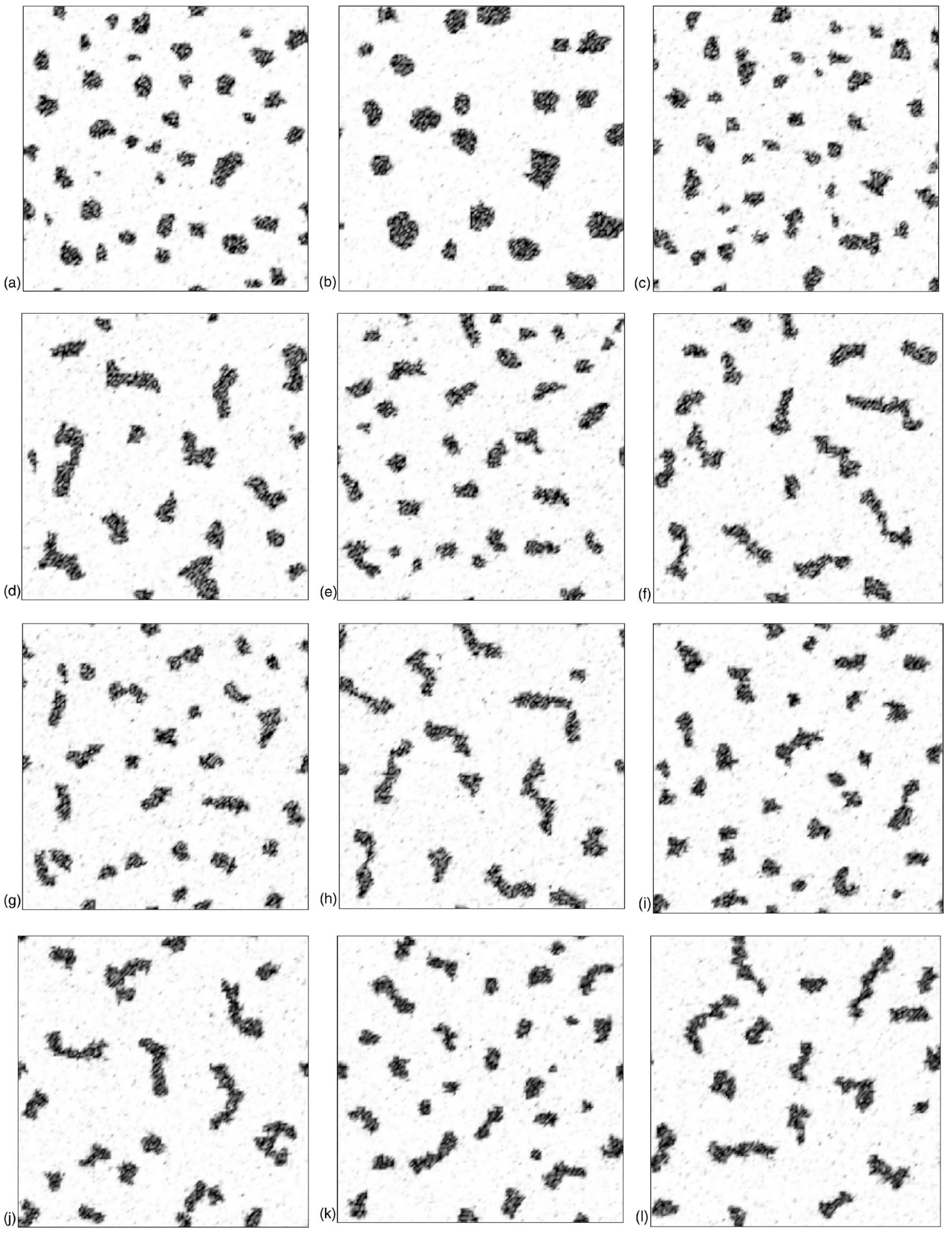

FIG. 11. Snapshots of the dynamics simulations for amphiphilic diblock copolymer solutions with various cutoff lengths $\lambda$. Black color represents $\phi_{B}(\boldsymbol{r})$. [(a) and (b)] $\lambda=1, \tilde{t}=312.5,1250$; [(c) and (d) $\lambda=2, \tilde{t}=312.5,1250$; [(e) and (f) $] \lambda=4, \tilde{t}=312.5,1250 ;[(\mathrm{g})$ and (h) $\lambda=5, \tilde{t}=312.5,1250 ;[(\mathrm{i})$ and (j) $\lambda \lambda=6, \tilde{t}$ $=312.5,1250$; and $[(\mathrm{k})$ and $(1)] \lambda=8, \tilde{t}=312.5,1250$. 


$$
\begin{aligned}
\left\langle\tilde{\xi}_{i}(\boldsymbol{r}, \widetilde{t}) \tilde{\xi}_{j}\left(\boldsymbol{r}^{\prime}, \widetilde{t}^{\prime}\right)\right\rangle \approx & -\frac{4 \widetilde{\beta}^{-1} \delta_{i j}}{\left|\boldsymbol{h}_{x}\right|\left|\boldsymbol{h}_{y}\right|\left|\boldsymbol{h}_{z}\right| \Delta \widetilde{t}} \sum_{\alpha=x, y, z} \frac{1}{\left|\boldsymbol{h}_{\alpha}\right|^{2}} \\
& {\left[\psi_{i}\left(\boldsymbol{r}+\boldsymbol{h}_{\alpha}\right) \psi_{i}(\boldsymbol{r})\left[\delta_{\left(\boldsymbol{r}+\boldsymbol{h}_{\alpha}\right) \boldsymbol{r}^{\prime}}-\delta_{\boldsymbol{r} \boldsymbol{r}^{\prime}}\right]\right.} \\
& -\psi_{i}(\boldsymbol{r}) \psi_{i}\left(\boldsymbol{r}-\boldsymbol{h}_{\alpha}\right) \\
& \left.\times\left[\delta_{\boldsymbol{r} \boldsymbol{r}^{\prime}}-\delta_{\left(\boldsymbol{r}-\boldsymbol{h}_{\alpha}\right) \boldsymbol{r}^{\prime}}\right]\right] \delta_{\tilde{t}^{\prime}} .
\end{aligned}
$$

In this work, we employ the following discretized equation to generate the noise field:

$$
\begin{aligned}
\widetilde{\xi}_{i}(\boldsymbol{r}, \widetilde{t}) \approx & 2 \sqrt{\widetilde{\beta}^{-1}} \sum_{\alpha=x, y, z} \frac{1}{\left|\boldsymbol{h}_{\alpha}\right|}\left[\sqrt{\psi_{i}\left(\boldsymbol{r}+\boldsymbol{h}_{\alpha}\right) \psi_{i}(\boldsymbol{r})} \widetilde{\omega}_{i}^{(\alpha)}(\boldsymbol{r}\right. \\
& \left.\left.+\boldsymbol{h}_{\alpha} / 2, \widetilde{t}\right)-\sqrt{\psi_{i}(\boldsymbol{r}) \psi_{i}\left(\boldsymbol{r}-\boldsymbol{h}_{\alpha}\right)} \tilde{\omega}_{i}^{(\alpha)}\left(\boldsymbol{r}-\boldsymbol{h}_{\alpha} / 2, \widetilde{t}\right)\right],
\end{aligned}
$$

where $\widetilde{\omega}_{i}^{(\alpha)}(\boldsymbol{r}, \widetilde{t})$ is the $\alpha$ element of $\widetilde{\boldsymbol{\omega}}_{i}(\boldsymbol{r}, \widetilde{t}) . \widetilde{\boldsymbol{\omega}}_{i}(\boldsymbol{r}, \widetilde{t})$ is the discretized Gaussian white noise field which satisfies

$$
\begin{aligned}
& \left\langle\widetilde{\boldsymbol{\omega}}_{i}(\boldsymbol{r}, \widetilde{t})\right\rangle=0, \\
& \left\langle\widetilde{\boldsymbol{\omega}}_{i}(\boldsymbol{r}, \widetilde{t}) \widetilde{\boldsymbol{\omega}}_{j}\left(\boldsymbol{r}^{\prime}, \widetilde{t}^{\prime}\right)\right\rangle=\frac{\delta_{i j} \delta_{r r^{\prime}} \delta_{\tilde{t}^{\prime}}}{\left|\boldsymbol{h}_{x}\right|\left|\boldsymbol{h}_{y}\right|\left|\boldsymbol{h}_{z}\right| \Delta \widetilde{t}} \mathbf{1} .
\end{aligned}
$$

Notice that $\widetilde{\boldsymbol{\omega}}_{i}(\boldsymbol{r}, \widetilde{t})$ is defined on the staggered lattice. The position of the staggered lattice point is $\boldsymbol{r}=\left(n_{x}+1 / 2\right) \boldsymbol{h}_{x}$ $+n_{y} \mathbf{h}_{y}+n_{z} \boldsymbol{h}_{z}, n_{x} \boldsymbol{h}_{x}+\left(n_{y}+1 / 2\right) \boldsymbol{h}_{y}+n_{z} \boldsymbol{h}_{z}, n_{x} \boldsymbol{h}_{x}+n_{y} \boldsymbol{h}_{y}+\left(n_{z}+1 / 2\right)$ $\times \boldsymbol{h}_{z}$, where $n_{\alpha}$ is the integer. It is easy to show that the noise field generated by Eq. (A9) satisfies Eq. (A8).

The noise generation scheme is finally written as follows.

(1) Generate the Gaussian white normal distribution vector noise field $\widetilde{\boldsymbol{\omega}}_{i}(\boldsymbol{r}, \widetilde{t})$ on the staggered lattice points. The noise is generated by using the Mersenne twister pseudo-random-number generator ${ }^{67}$ and the standard Box-Muller method. ${ }^{46}$

(2) Calculate the noise field $\widetilde{\xi}_{i}(\boldsymbol{r}, \widetilde{t})$ from $\psi_{i}(\boldsymbol{r})$ and $\widetilde{\boldsymbol{\omega}}_{i}(\boldsymbol{r}, \widetilde{t})$ by using Eq. (A9).

\section{APPENDIX B: FINITE SIZE EFFECT}

It is well known that the size of the simulation box often affects the simulation results (the finite size effect). In this appendix, the finite size effect for the simulations is studied by performing simulations with different box sizes.

Figure 10 shows the simulation results for different (small) box sizes. All the parameters except for the box size are the same for the previous vesicle formation simulation (Fig. 2). The box sizes are set to $6 b \times 6 b \times 6 b(12 \times 12$ $\times 12$ lattice points), $8 b \times 8 b \times 8 b \quad(16 \times 16 \times 16$ lattice points), $12 b \times 12 b \times 12 b(24 \times 24 \times 24$ lattice points $)$, and $16 b \times 16 b \times 16 b(32 \times 32 \times 32$ lattice points). The spherical micelles [Fig. 10(a)] and the cylindrical micelles [Figs. 10(b) and 10(c)] for small boxes can be observed. These structures are much different from the vesicle structures in Fig. 10(d) or Fig. 2. This means that if the simulation box is too small, vesicles cannot be obtained.
It also implies that there may be the finite size effect in the vesicle formation simulations (box size of $48 \times 48 \times 48$ ), since the sizes of obtained vesicles are in most cases comparable to the box sizes. But at least one can claim that the vesicle formation process itself is not affected qualitatively since the system size is not so small for the formation of disklike structures [unlike the case of Figs. 10(a)-10(c)], and one can observe that the small vesicle formation process [Fig. 3(d)] is qualitatively the same as for larger vesicles.

\section{APPENDIX C: CUTOFF LENGTH DEPENDENCY}

The cutoff length $\lambda$ for the long range interaction term in Eq. (3) is introduced rather intuitively, and therefore the value of $\lambda$ for simulations should be considered carefully. In this section, the simulations with different values of $\lambda$ are performed. Simulations are performed in two dimensions. Parameters are as follows: $N=10, f_{A}=1 / 3$, and $f_{B}=2 / 3, \lambda$ $=2,4,5,6,8 ; \bar{\phi}_{S}=1-\bar{\phi}_{p}=0.8 ; \chi_{A B}=2.5, \chi_{A S}=-0.5, \chi_{B S}=5$, and $b=1$. The size of the two-dimensional simulation box is $64 b \times 64 b(128 \times 128$ lattice points, periodic boundary condition). The time steps are $\Delta \tilde{t}=0.0025$ (for $\lambda=2,4,5$ ), 0.000625 (for $\lambda=6$ ), and $\Delta \tilde{t}=0.0003125$ (for $\lambda=8$ ). The magnitude of the noise is $\widetilde{\beta}^{-1}=0.078125$. All the simulations are started from the homogeneous state.

Figure 11 shows the results for various value of the cutoff length ([(a) and (b) $\lambda=1,[(\mathrm{c})$ and (d) $] \lambda=2,[(\mathrm{e})$ and (f) $]$ $\lambda=4,[(\mathrm{~g})$ and $(\mathrm{h})] \lambda=5,[(\mathrm{i})$ and $(\mathrm{j})] \lambda=6$, and [(k) and (l)] $\lambda=8)$ at $\tilde{t}=312.5,1250$. One can see that the dropletlike structures are observed for $\lambda=1$ and micellar structures for $2 \leqslant \lambda \leqslant 8$. The resulting morphologies for $2 \leqslant \lambda \leqslant 8$ are qualitatively the same. (Strictly speaking, $\lambda=2$ looks slightly different from others and somehow resembles to $\lambda=1$.) As expected, the cutoff length dependence of the morphology is small. Thus even if the value of $\lambda$ is not accurate, the results are expected not to be affected qualitatively. From this result, one can justify to use the value $\lambda=5$, which is estimated by the rough argument.

${ }^{1}$ D. E. Discher and A. Eisenberg, Science 297, 967 (2002).

${ }^{2}$ A. Choucair and A. Eisenberg, Eur. Phys. J. E 10, 37 (2003).

${ }^{3}$ H. Noguchi and M. Takasu, Phys. Rev. E 64, 041913 (2001).

${ }^{4}$ S. Yamamoto, Y. Maruyama, and S. Hyodo, J. Chem. Phys. 116, 5842 (2002).

${ }^{5}$ S. Marrink and A. E. Mark, J. Am. Chem. Soc. 125, 15233 (2003).

${ }^{6}$ A. T. Bernardes, J. Phys. II 6, 169 (1996).

${ }^{7}$ J. Leng, S. U. Egelhaaf, and M. E. Cates, Europhys. Lett. 59, 311 (2002).

${ }^{8}$ J. Leng, S. U. Egelhaaf, and M. E. Cates, Biophys. J. 85, 1624 (2003).

${ }^{9}$ L. Chen, H. Shen, and A. Eisenberg, J. Phys. Chem. B 103, 9488 (1999).

${ }^{10}$ L. Leibler, Macromolecules 13, 1602 (1980).

${ }^{11}$ T. Ohta and K. Kawasaki, Macromolecules 19, 2621 (1986).

${ }^{12}$ Y. Bohbot-Raviv and Z.-G. Wang, Phys. Rev. Lett. 85, 3428 (2000).

${ }^{13}$ M. W. Matsen and M. Schick, Phys. Rev. Lett. 72, 2660 (1994).

${ }^{14}$ M. W. Matsen and F. S. Bates, Macromolecules 29, 1091 (1996).

15 J. G. E. M. Fraaije, J. Chem. Phys. 99, 9202 (1993).

${ }^{16}$ G. H. Fredrickson, V. Ganesan, and F. Drolet, Macromolecules 35, 16 (2002).

${ }^{17}$ X. He, H. Liang, L. Huang, and C. Pan, J. Phys. Chem. B 108, 1731 (2004).

${ }^{18}$ T. Uneyama and M. Doi, Macromolecules 38, 5817 (2005).

${ }^{19}$ G. J. A. Sevink and A. V. Zvelindovsky, Macromolecules 38, 7502 (2005).

${ }^{20}$ S. Koizumi, H. Hasegawa, and T. Hashimoto, Macromolecules 27, 6532 (1994). 
${ }^{21}$ T. Ohta and A. Ito, Phys. Rev. E 52, 5250 (1995).

${ }^{22}$ T. Uneyama and M. Doi, Macromolecules 38, 196 (2005).

${ }^{23}$ J. G. E. M. Fraaije and G. J. A. Sevink, Macromolecules 36, 7891 (2003).

${ }^{24}$ X. He and F. Schmid, Macromolecules 39, 2654 (2006).

${ }^{25}$ N. M. Maurits and J. G. E. M. Fraaije, J. Chem. Phys. 107, 5879 (1997).

${ }^{26}$ M. Bahiana and Y. Oono, Phys. Rev. A 41, 6763 (1990).

${ }^{27}$ T. Kawakatsu, Statistical Physics of Polymers: An Introduction (Springer, Berlin, 2004).

${ }^{28}$ N. M. Maurits and J. G. E. M. Fraaije, J. Chem. Phys. 106, 6730 (1997).

${ }^{29}$ P. G. de Gennes, J. Chem. Phys. 72, 4756 (1980).

${ }^{30}$ A. Y. Grosberg and A. R. Khokhlov, Statistical Physics of Macromolecules (American Institute of Physics, New York, 1994).

${ }^{31}$ H. Frusawa, J. Phys.: Condens. Matter 17, L241 (2005).

${ }^{32}$ A. A. Louis, P. G. Bolhuis, J. P. Hansen, and E. J. Meijer, Phys. Rev. Lett. 85, 2522 (2000).

${ }^{33}$ I. Pagonabarraga and M. E. Cates, Europhys. Lett. 55, 348 (2001).

${ }^{34}$ H. Tang and K. F. Freed, J. Chem. Phys. 97, 4496 (1992).

${ }^{35} \mathrm{~T}$. Uneyama (unpublished).

${ }^{36}$ F. Drolet and G. H. Fredrickson, Phys. Rev. Lett. 83, 4381 (1999).

${ }^{37}$ I. M. Lifshizt, A. Y. Grosberg, and A. R. Khokhlov, Rev. Mod. Phys. 50, 683 (1978).

${ }^{38}$ T. Hashimoto, M. Shibayama, and H. Kawai, Macromolecules 13, 1237 (1980).

${ }^{39}$ D. S. Dean, J. Phys. A 29, L613 (1996).

${ }^{40}$ H. Frusawa and R. Hayakawa, J. Phys. A 33, L155 (2000).

${ }^{41}$ A. J. Archer and M. Rauscher, J. Phys. A 37, 9325 (2004).

${ }^{42}$ B. A. C. van Vlimmeren and J. G. E. M. Fraaije, Comput. Phys. Commun. 99, 21 (1996).

${ }^{43}$ J. S. Langer, M. Bar-on, and H. D. Miller, Phys. Rev. A 11, 1417 (1975).

${ }^{44}$ K. Kitahara and M. Imada, Suppl. Prog. Theor. Phys. 64, 65 (1978).

${ }^{45}$ M. Doi and S. F. Edwards, The Theory of Polymer Dynamics (Oxford University Press, Oxford, 1986).

${ }^{46}$ W. H. Press, S. A. Teukolsky, W. T. Vetterling, and B. P. Flannery, $\mathrm{Nu}-$ merical Recipes in $C$, 2nd ed. (Cambridge University Press, Cambridge, 1992).

${ }^{47}$ M. Frigo and S. G. Johnson, Proc. IEEE 93, 216 (2005); http:// www.fftw.org/

${ }^{48}$ A. Shinozaki and Y. Oono, Phys. Rev. E 48, 2622 (1993).

${ }^{49}$ H. Shen and A. Eisenberg, J. Phys. Chem. B 103, 9473 (1999).

${ }^{50}$ H. Shen and A. Eisenberg, Macromolecules 33, 2561 (2000).

${ }^{51}$ P. Bhargava, J. X. Zheng, P. Li, R. P. Quirk, F. W. Harris, and S. Z. D. Cheng, Macromolecules 39, 4880 (2006).

${ }^{52}$ C.-Z. Zhang and Z.-G. Wang, Phys. Rev. E 73, 031804 (2006).

${ }^{53}$ A. V. M. Zvelindovsky, B. A. C. van Vlimmeren, G. J. A. Sevink, N. M. Maurits, and J. G. E. M. Fraaije, J. Chem. Phys. 109, 8751 (1998).

${ }^{54}$ A. V. Zvelindovsky, G. J. A. Sevink, B. A. C. van Vlimmeren, N. M. Maurits, and J. G. E. M. Fraaije, Phys. Rev. E 57, R4879 (1998).

${ }^{55}$ B. A. C. van Vlimmeren, N. M. Maurits, A. V. Zvelindovsky, G. J. A. Sevink, and J. G. E. M. Fraaije, Macromolecules 32, 646 (1999).

${ }^{56}$ B. A. C. van Vlimmeren, M. Postma, P. Huetz, A. Brisson, and J. G. E. M. Fraaije, Phys. Rev. E 54, 5836 (1999).

${ }^{57}$ E. E. Dormidontova, Macromolecules 35, 987 (2002).

${ }^{58}$ Y.-M. Lam and G. Goldbeck-Wood, Polymer 44, 3593 (2003).

${ }^{59}$ R. Xu, M. A. Winnik, G. Riess, B. Chu, and M. D. Croucher, Macromolecules 25, 644 (1992).

${ }^{60}$ L. Zhu, S. Z. D. Cheng, B. H. Calhoun, Q. Ge, R. P. Quirk, E. L. Thomas, B. S. Hsiao, F. Yeh, and B. Lotz, Polymer 42, 5829 (2001).

${ }^{61} \mathrm{H}$. Watanabe and T. Kotaka, Macromolecules 20, 530 (1986).

${ }^{62}$ PoLyInfo, http://polymer.nims.go.jp/

${ }^{63}$ G. D. Wignall, D. G. H. Ballard, and J. Schelten, Eur. Polym. J. 10, 861 (1974).

${ }^{64}$ M. Müller and F. Schmid, Adv. Polym. Sci. 185, 1 (2005).

${ }^{65}$ R. D. Groot and P. B. Warren, J. Chem. Phys. 107, 4423 (1997).

${ }^{66}$ T. Honda and T. Kawakatsu, Macromolecules 40, 1227 (2007).

${ }^{67}$ M. Matsumoto and T. Nishimura, ACM Trans. Model. Comput. Simul. 8, 3 (1998); http://www.math.sci.hiroshima-u.ac.jp/ m-mat/MT/emt.html 